Research Article

\title{
Stochastic Periodic Solution and Permanence of a Holling-Leslie Predator-Prey System with Impulsive Effects
}

\author{
Jinxing Zhao $\mathbb{D}^{1}$ and Yuanfu Shao $\mathbb{D}^{2}$ \\ ${ }^{1}$ School of Mathematical Sciences, Inner Mongolia University, Hohhot 010021, Inner Mongolia, China \\ ${ }^{2}$ College of Science, Guilin University of Technology, Guilin 541004, Guangxi, China \\ Correspondence should be addressed to Jinxing Zhao; zhjxing@imu.edu.cn and Yuanfu Shao; shaoyuanfu@163.com
}

Received 16 October 2020; Revised 11 December 2020; Accepted 18 December 2020; Published 5 January 2021

Academic Editor: Yongqiang Fu

Copyright (c) 2021 Jinxing Zhao and Yuanfu Shao. This is an open access article distributed under the Creative Commons Attribution License, which permits unrestricted use, distribution, and reproduction in any medium, provided the original work is properly cited.

Considering the environmental effects, a Holling-Leslie predator-prey system with impulsive and stochastic disturbance is proposed in this paper. Firstly, we prove that existence of periodic solution, the mean time boundness of variables is found by integral inequality, and we establish some sufficient conditions assuring the existencle of periodic Markovian process. Secondly, for periodic impulsive differential equation and system, it is different from previous research methods, by defining three restrictive conditions, we study the extinction and permanence in the mean of all species. Thirdly, by stochastic analysis method, we investigate the stochastic permanence of the system. Finally, some numerical simulations are given to illustrate the main results.

\section{Introduction}

Predator-prey phenomenon is very popular in natural world, and recently more and more researchers pay attention to investigate the complicated dynamical behaviors between predator and prey species, which has been and is long to be one of the most important hot topics in the future [1]. Hsu and Huang [2] proposed the following Holling-Tanner model:

$$
\left\{\begin{array}{l}
\mathrm{d} x(t)=x(t)\left(a_{1}-b_{1} x(t)-\frac{a y(t)}{b+x(t)}\right) \mathrm{d} t \\
\mathrm{~d} y(t)=y(t)\left(a_{2}-b_{2} \frac{y(t)}{x(t)}\right) \mathrm{d} t
\end{array}\right.
$$

where $x(t)$ and $y(t)$ are the densities of prey and predator at time $t$, respectively; $a_{i}(i=1,2)$ is the intrinsic growth rate of prey or predator; and $b_{1}$ is the density-dependent coefficient; $(a y(t) / b+x(t))$ represents Holling type II functional response, where $a, b>0$ denote the capturing rate and half capturing saturation constant, respectively. Function $\left(b_{2} y(t) / x(t)\right)$ is the Leslie-Gower term, which measures the loss in the predator population due to rarity of its favorite food, where $\left(a_{2} x / b_{2}\right)$ is the carrying capacity. For more biological meanings of this model, see [3-5].

Moreover, in natural world, there are many kinds of functional responses. If the functional response is Holling type IV, then the model reads

$$
\left\{\begin{array}{l}
\mathrm{d} x(t)=x(t)\left(a_{1}-b_{1} x(t)-\frac{a y(t)}{b+x^{2}(t)}\right) \mathrm{d} t \\
\mathrm{~d} y(t)=y(t)\left(a_{2}-b_{2} \frac{y(t)}{x(t)}\right) \mathrm{d} t .
\end{array}\right.
$$

Its dynamics has been sufficiently studied, and many better results have been obtained by $\mathrm{Li}$ and Xiao [6].

On the contrary, in practice, the environmental white noise almost exists everywhere. For ecological system, the growth rate of population is inevitably affected by the white noise. In order to reveal the effect of white noise, random disturbance is introduced in many mathematical models [7-12]. Meanwhile, due to the individual life cycle and 
seasonal variation and so on, the birth rate, death rate, carrying capacity of species, and other parameters always exhibit cycle changes [13-15]. Therefore, Jiang et al. [16] proposed the following nonautonomous stochastic model:

$$
\left\{\begin{array}{l}
\mathrm{d} x(t)=x(t)\left(a_{1}(t)-b_{1}(t) x(t)-\frac{a(t) y(t)}{b(t)+x^{n}(t)}\right) \mathrm{d} t+\sigma_{1}(t) x(t) \mathrm{d} B_{1}(t), \\
\mathrm{d} y(t)=y(t)\left(a_{2}(t)-b_{2}(t) \frac{y(t)}{x(t)}\right) \mathrm{d} t+\sigma_{2}(t) y(t) \mathrm{d} B_{2}(t),
\end{array}\right.
$$

where $a_{i}(t), b_{i}(t), \sigma_{i}(t)(i=1,2), a(t)$, and $b(t)$ are all positive $T$-periodic functions; $B_{1}(t)$ and $B_{2}(t)$ are independent standard Brownian motions defined on the probability space $\left(\Omega, F,\left\{F_{t}\right\}_{t \geq 0}, P\right)$ with a filtration $\left\{F_{t}\right\}_{t \geq 0}$ satisfying the usual conditions (i.e., it is right continuous, and $F_{0}$ contains all $p$-null set); and $\sigma_{i}^{2}(i=1,2)$ denotes the density of white noise. Parameter $n=1$ or $n=2$ means Holling type II or Holling type IV functional response, respectively.

However, for population system, the effect from natural or man-made factors is very popular, and hence, the growth of species will have to suffer from some discrete changes of relatively short time interval at some periodic times, such as stocking and harvesting. These effects are often modeled by impulsive parameters. In the last decades, many impulsive dynamical systems have been proposed and many better results have been reported, see, [17-23] and references therein. For example, the authors Zuo and Jiang [23] investigated the periodic solution and boundary periodic solution of the following impulsive model:

$$
\left\{\begin{array}{l}
\mathrm{d} x(t)=x(t)\left(a_{1}(t)-b_{1}(t) x(t)-\frac{a(t) y(t)}{b(t)+x(t)}\right) \mathrm{d} t+\sigma_{1}(t) x(t) \mathrm{d} B_{1}(t) \\
\mathrm{d} y(t)=y(t)\left(a_{2}(t)-b_{2}(t) \frac{y(t)}{x(t)}\right) \mathrm{d} t+\sigma_{2}(t) y(t) \mathrm{d} B_{2}(t) \\
x_{1}\left(t_{k}^{+}\right)=\left(1+\xi_{1 k}\right) x_{1}\left(t_{k}\right) \\
x_{2}\left(t_{k}^{+}\right)=\left(1+\xi_{2 k}\right) x_{2}\left(t_{k}\right)
\end{array}\right\}, \quad t=t_{k} .
$$

Inspired by the above discussion, considering some natural or man-made impulsive factors, we propose the following stochastic predator-prey model:

$$
\left\{\begin{array}{l}
\mathrm{d} x(t)=x(t)\left(a_{1}(t)-b_{1}(t) x(t)-\frac{a(t) y(t)}{b(t)+x^{n}(t)}\right) \mathrm{d} t+\sigma_{1}(t) x(t) \mathrm{d} B_{1}(t) \\
\mathrm{d} y(t)=y(t)\left(a_{2}(t)-b_{2}(t) \frac{y(t)}{x(t)}\right) \mathrm{d} t+\sigma_{2}(t) y(t) \mathrm{d} B_{2}(t) \\
x_{1}\left(t_{k}^{+}\right)=\left(1+\xi_{1 k}\right) x_{1}\left(t_{k}\right) \\
x_{2}\left(t_{k}^{+}\right)=\left(1+\xi_{2 k}\right) x_{2}\left(t_{k}\right)
\end{array}\right\}, \quad t=t_{k},
$$


where all coefficients $a_{1}(t), a_{2}(t), b_{1}(t), b_{2}(t)$, $a(t), b(t), \sigma_{1}(t)$, and $\sigma_{2}(t)$ are bounded, continuous, and periodic with period $T$. The impulsive points satisfy $0<t_{1}<t_{2}<\cdots, \lim _{k \longrightarrow \infty} t_{k}=+\infty$, and there exists an integer $p$ such that $t_{k+p}=t_{k}+T, \lambda_{i, k+p}=\lambda_{i k}, i=1,2$. Furthermore, by the biological meanings, we assume $\xi_{i k}>-1$ for $i=1,2$. For the biological meanings of all parameters, refer to $[2,6,16,23]$.

Our main aim of this paper is as follows: firstly, for determinate system, the existence of equilibrium or periodic solution is an important topic for the dynamics of biological system [24-27]. Similarly, for stochastic system, it is very interesting to study whether there exists a periodic Markovian process or not.

Secondly, for predator-prey system, the dynamical behaviors are another important topic [28-30]. By the comparison method, we establish some sufficient conditions assuring the extinction, permanence in the mean of all species, and the stochastic permanence of system (5).

The rest of the work of this paper is organized as follows: Section 2 begins with some notations, definitions, and important lemmas. Section 3 is devoted to the existence and uniqueness of the periodic Markovian process. Section 4 focuses on the extinction and permanence in the mean of species. Section 5 focuses on the stochastic permanence of system (5). Some numerical simulations are given to verify our main results in Section 6. Finally, we conclude this paper with a brief conclusion and discussion in Section 7.

\section{Preliminaries}

For an $n$-dimensional stochastic differential equation [13],

$$
\mathrm{d} x(t)=f(x(t), t) \mathrm{d} t+g(x(t), t) \mathrm{d} B(t), \quad t \geq t_{0},
$$

with initial value $x\left(t_{0}\right)=x_{0} \in R^{n}$, where $B(t)$ is an $n$-dimensional standard Brownian motion. The differential operator $L$ associated (6) is defined by

$$
L=\frac{\partial}{\partial t}+\sum_{k=1}^{n} f_{k}(x, t) \frac{\partial}{\partial x_{k}}+\frac{1}{2} \sum_{k, j=1}^{n}\left[g^{T}(x, t) g(x, t)\right]_{k j} \frac{\partial^{2}}{\partial x_{k} \partial x_{j}} \text {. }
$$

For bounded and continuous function $f(t)$, set

$$
\begin{aligned}
f^{u} & =\sup _{t \geq 0} f(t), \\
f^{l} & =\inf _{t \geq 0} f(t), \\
f^{*} & =\limsup _{t \longrightarrow+\infty} f(t), \\
f_{*} & =\liminf _{t \longrightarrow+\infty} f(t),
\end{aligned}
$$

and if $f(t)$ is integrable, then define

$$
\begin{aligned}
\langle f\rangle & =\frac{1}{t} \int_{0}^{t} f(s) \mathrm{d} s, \\
\langle f\rangle_{T} & =\frac{1}{T} \int_{0}^{T} f(s) \mathrm{d} s .
\end{aligned}
$$

To investigate the dynamics of (5), we consider the following nonimpulsive system:

$$
\left\{\begin{array}{l}
\mathrm{d} X(t)=X(t)\left(a_{1}(t)+\frac{1}{T} \sum_{j=1}^{p} \ln \left(1+\xi_{1 k}\right)-b_{1}(t) A_{1}(t) X(t)-\frac{a(t) A_{2}(t) Y(t)}{b(t)+\left(A_{1}(t) X(t)\right)^{n}}\right) \mathrm{d} t+\sigma_{1}(t) X(t) \mathrm{d} B_{1}(t) \\
\mathrm{d} Y(t)=Y(t)\left(a_{2}(t)+\frac{1}{T} \sum_{j=1}^{p} \ln \left(1+\xi_{2 k}\right)-b_{2}(t) \frac{A_{2}(t) Y(t)}{A_{1}(t) X(t)}\right) \mathrm{d} t+\sigma_{2}(t) Y(t) \mathrm{d} B_{2}(t)
\end{array}\right.
$$

where

$$
\begin{aligned}
& A_{1}(t)=\left[\prod_{j=1}^{p}\left(1+\xi_{1 j}\right)\right]^{-(t / T)} \prod_{0 \leq t_{k}<t}\left(1+\xi_{1 k}\right), \\
& A_{2}(t)=\left[\prod_{j=1}^{p}\left(1+\xi_{2 j}\right)\right]^{-(t / T)} \prod_{0 \leq t_{k}<t}\left(1+\xi_{2 k}\right) .
\end{aligned}
$$

It is easy to show that $A_{1}(t)$ and $A_{2}(t)$ both are periodic functions with period $T$ (for details, see [27]). We assume the product equals unity if the number of factors is zero and $\varepsilon$ stands for a sufficiently small positive constant whose value may be different at different places.

Now we present the definitions of periodic Markovian process and the solution of impulsive stochastic differential equation, and some auxiliary results of the existence of periodic Markovian process.

Definition 1 (see $[13,27])$. A stochastic process $\xi(t)=\xi(t, \omega)(-\infty<t<\infty)$ is said to be periodic with periodic $T$ if for every finite sequence of numbers $t_{1}, t_{2}, \ldots, t_{n}$, the joint distribution of random variables $\xi\left(t_{1}+h\right), \ldots, \xi\left(t_{n}+h\right)$ is independent of $h$, where $h=k T, k= \pm 1, \pm 2, \ldots$.

Definition 2 (see [22, 27]). System (5) is stochastically permanent if for every $\varepsilon \in(0,1)$, there is a pair of constants $\alpha>0$ and $\beta>0$ such that for any initial data $(x(0), y(0)) \in R_{+}^{2}$, the solution $(x(t), y(t))$ of (5) has the property that 


$$
\begin{aligned}
& \liminf _{t \longrightarrow+\infty} P\{|(x(t), y(t))| \geq \alpha\} \geq 1-\varepsilon, \\
& \limsup _{t \longrightarrow+\infty} P\{|(x(t), y(t))| \leq \beta\} \geq 1-\varepsilon,
\end{aligned}
$$

where $P$ represents the probability of the events.

Definition 3 (see [20, 22]). For the following impulsive stochastic differential equation

$$
\left\{\begin{array}{l}
\mathrm{d} x(t)=f(t, x(t)) \mathrm{d} t+g(t, x(t)) \mathrm{d} B(t), \quad t \neq t_{k}, \\
x\left(t_{k}^{+}\right)=\left(1+\lambda_{k}\right) x\left(t_{k}\right), \quad k \in N,
\end{array}\right.
$$

with initial value $x(0)=x_{0}$, a stochastic process $x(t)=\left(x_{1}(t), x_{2}(t), \ldots, x_{n}(t)\right)^{T}, \quad t \in R_{+}$, is said to be a solution of the above system, if

(i) $x(t)$ is $f_{t}$ adapted and is continuous on $\left(0, t_{1}\right)$ and each interval $\left(t_{k}, t_{k+1}\right) \subset R_{+}, k \in N, f(t, x(t))$ $\in L\left(R_{+}, R^{n}\right)$, and $g(t, x(t)) \in L^{2}\left(R_{+}, R^{n}\right)$, where $L^{p}\left(R_{+}, R^{n}\right)$ is all $R^{n}$-valued measurable $f_{t}$-adapted processes $f(t)$ satisfying $\int_{0}^{T}|f(t)|^{p} \mathrm{~d} t<+\infty$ almost surely for all $T>0, p=1,2$.

(ii) For every $t_{k}, k \in N, \quad x\left(t_{k}^{+}\right)=\lim _{t \longrightarrow t_{k}^{+}} x(t)$ and $x\left(t_{k}^{-}\right)=\lim _{t \longrightarrow t_{k}^{-}} x(t)$ exist, and $x\left(t_{k}\right)={ }^{k} x\left(t_{k}^{-}\right)$with probability one.

(iii) For all $t \in\left[0, t_{1}\right], x(t)$ obeys the integral equation

$$
x(t)=x(0)+\int_{0}^{t} f(s, x(s)) \mathrm{d} s+\int_{0}^{t} g(s, x(s)) \mathrm{d} B(s),
$$

and for all $t \in\left[t_{k}, t_{k+1}\right], k \in N, x(t)$ obeys the following integral equation:

$$
x(t)=x\left(t_{k}^{+}\right)+\int_{t_{k}}^{t} f(s, x(s)) \mathrm{d} s+\int_{t_{k}}^{t} g(s, x(s)) \mathrm{d} B(s) .
$$

Lemma 1 (see $[13,27])$. For the following Itô's differential equation

$$
\mathrm{d} x(t)=b(t, x(t)) \mathrm{d} t+\sigma(t, x(t)) \mathrm{d} B(t),
$$

if all the coefficients are $T$-periodic in $t$ and satisfy the linear growing condition and the Lipschitz condition in every cylinder $U_{l} \times R_{+}$for $l>0$, where $U_{l}=\{x:\|x\| \leq l\}$ and there exists a function $v=v(t, x)$ which is twice continuously differentiable with respect to $x$ and once continuously differentiable with respect to $t$ in $R^{n} \times R_{+}, T$ is periodic in $t$ and satisfies the following conditions:

$$
\begin{gathered}
\inf _{\|x\|>l} v(t, x) \longrightarrow+\infty, \quad \text { as } l \longrightarrow+\infty \\
L v(t, x) \leq-1 \text { outside some compact set, }
\end{gathered}
$$

then there exists a solution of (16) which is $T$-periodic Markovian process.

Lemma 2 (see [20]). Suppose that $Z(t) \epsilon$ $C\left[\Omega \times[0,+\infty), R_{+}\right]$and $\lim _{t \longrightarrow+\infty} F(t) / t=0$, a.s.

(a) If there exist two positive constants $T>0, \lambda_{0}>0$ such that, for all $t>T$,

$$
\ln Z(t) \leq \lambda t-\lambda_{0} \int_{0}^{t} z(s) \mathrm{d} s+F(t), \quad \text { a.s }
$$

then

$$
\begin{cases}\langle Z\rangle^{*} \leq \frac{\lambda}{\lambda_{0}}, & \text { a.s. if } \quad \lambda \geq 0, \\ \lim _{t \rightarrow+\infty} Z(t)=0, & \text { a.s. if } \quad \lambda<0 .\end{cases}
$$

(b) If there exist some constants $T>0, \lambda_{0}>0, \lambda$ such that, for all $t>T$

$$
\ln Z(t) \geq \lambda t-\lambda_{0} \int_{0}^{t} z(s) \mathrm{d} s+F(t), \quad a . s,
$$

then 


$$
\langle Z\rangle_{*} \geq \frac{\lambda}{\lambda_{0}}, \quad \text { a.s. }
$$

Lemma 3. Let $x(t)=A_{1}(t) X(t), y(t)=A_{2}(t) Y(t)$; then

(1) if $(x(t), y(t))$ is the solution of (5), then $(X(t), Y(t))$ is the solution of (10).

(2) if $(X(t), Y(t))$ is the solution of (10), then $(x(t), y(t))$ is the solution of (5).

Remark 1. The proof is similar with that of [20] and is omitted. Lemma 3 shows that the dynamics of (5) is equivalent to that of (10). Hence, in the later, we mainly consider (10) to reveal the dynamical properties of (5).

As to the existence of nontrivial positive solution of (5), we have Lemma 4.

Lemma 4. For any given initial value $(x(0), y(0)) \in R_{+}^{2}$, system (5) has a unique solution $(x(t), y(t))$ on $t \geq 0$, and the solution remains in $R_{+}^{2}$ with probability one.

Remark 2. Lemma 3 implies that the existence of solution $(x(t), y(t))$ of $(5)$ on $t \geq 0$ is equivalent to the existence of the solution $(X(t), Y(t))$ of $(10)$. The proof of the existence of $(X(t), Y(t))$ of $(10)$ is similar with that of [23] and is omitted here.

\section{Existence of Periodic Solution}

In this section, we focus on the existence of periodic Markovian process of (5). Above all, we give the following assumption.

Theorem 1. Suppose the following condition holds,

$$
\begin{aligned}
\left(H_{1}\right) \zeta & :=\left\langle a_{1}(t)-\sigma_{1}^{2}(t)\right\rangle_{T}+\frac{1}{T} \sum_{j=1}^{p} \ln \left(1+\xi_{1 k}\right)>0, \\
\eta & :=\left\langle a_{2}(t)-\sigma_{2}^{2}(t)\right\rangle_{T}+\frac{1}{T} \sum_{j=1}^{p} \ln \left(1+\xi_{2 k}\right)>0,
\end{aligned}
$$

then there exists a solution for system (5), which is a T-periodic Markovian process.

Proof. According to the equivalent property and existence of solutions (Lemmas 3 and 4), we only need to prove that, under $\left(H_{1}\right)$, the solution of system (10) is a periodic Markovian process. Lemma 1 shows that it suffices to find a $C^{2}$-function $V(t, X, Y)$ and a closed set $U \in R_{+}^{2}$ such that all conditions of Lemma 1 hold. Define

$$
\begin{aligned}
& V_{1}(t, X)=\frac{e^{\mu_{1}(t)}}{X}+\ln X, \\
& V_{2}(t, Y)=\frac{q e^{\mu_{2}(t)}}{Y}+c \ln Y,
\end{aligned}
$$

where $q$ and $c$ are two constants defined later and $\mu_{1}(t)$ and $\mu_{2}(t)$ are the positive continuous function such that

$$
\begin{aligned}
& \mu_{1}^{\prime}(t)=a_{1}(t)-\sigma_{1}^{2}(t)-\left\langle a_{1}(t)-\sigma_{1}^{2}(t)\right\rangle_{T}, \\
& \mu_{2}^{\prime}(t)=a_{2}(t)-\sigma_{2}^{2}(t)-\left\langle a_{2}(t)-\sigma_{2}^{2}(t)\right\rangle_{T} .
\end{aligned}
$$

It is not difficult to verify that $\mu_{i}(t)(i=1,2)$ is $T$-periodic on $[0, \infty)$, and

$$
\liminf _{(X, Y) \in R_{+}^{2} / U_{k}} V(t, X, Y) \longrightarrow \infty, \quad \text { as } k \longrightarrow \infty,
$$

where $U_{k}=\{(X, Y):(X, Y) \in((1 / k), k) \times((1 / k), k)\}$. Hence $V(t, X, Y)$ is $T$-periodic and satisfies the first condition of Lemma 1. Applying the Itô's formula to $V_{1}(t, X)$ and $V_{2}(t, Y)$, then 


$$
\begin{aligned}
& \operatorname{LV}_{1}(t, X)=\frac{e^{\mu_{1}(t)}}{X}\left(\mu_{1}^{\prime}(t)-a_{1}(t)-\frac{1}{T} \sum_{j=1}^{p} \ln \left(1+\xi_{1 k}\right)+\sigma_{1}^{2}(t)\right) \\
& +a_{1}(t)+\frac{1}{T} \sum_{j=1}^{p} \ln \left(1+\xi_{1 k}\right)-b_{1}(t) A_{1}(t) X-\frac{a(t)) A_{2}(t) Y}{b(t)+\left(A_{1}(t) X\right)^{n}} \\
& +e^{\mu_{1}(t)} b_{1}(t) A_{1}(t)+\frac{e^{\mu_{1}(t)}}{X} \frac{a(t) A_{2}(t) Y}{b(t)+\left(A_{1}(t) X\right)^{n}}-\frac{\sigma_{1}^{2}(t)}{2} \\
& =a_{1}(t)+\frac{1}{T} \sum_{j=1}^{p} \ln \left(1+\xi_{1 k}\right)-\frac{\sigma_{1}^{2}(t)}{2}+e^{\mu_{1}(t)} b_{1}(t) A_{1}(t)-b_{1}(t) A_{1}(t) X \\
& +\frac{e^{\mu_{1}(t)}}{X}\left(\mu_{1}^{\prime}(t)-a_{1}(t)-\frac{1}{T} \sum_{j=1}^{p} \ln \left(1+\xi_{1 k}\right)+\sigma_{1}^{2}(t)\right) \\
& +\frac{e^{\mu_{1}(t)}}{X} \frac{a(t) A_{2}(t) Y}{b(t)+\left(A_{1}(t) X\right)^{n}}-\frac{a(t) A_{2}(t) Y}{b(t)+\left(A_{1}(t) X\right)^{n}} \\
& \leq a_{1}^{u}+\frac{1}{T} \sum_{j=1}^{p} \ln \left(1+\xi_{1 k}\right)-\frac{\left(\sigma_{1}^{2}\right)^{l}}{2}+e^{\left|\mu_{1}^{u}\right|} b_{1}^{u} A_{1}^{u}-b_{1}^{l} A_{1}^{l} X \\
& -\frac{e^{\mu_{1}(t)}}{X}\left(\left\langle a_{1}(t)-\sigma_{1}^{2}(t)\right\rangle_{T}+\frac{1}{T} \sum_{j=1}^{p} \ln \left(1+\xi_{1 k}\right)\right) \\
& +\frac{a^{u} A_{2}^{u} e^{\left|\mu_{1}^{u}\right|} Y}{b^{l} X}-\frac{a^{l} A_{2}^{l} Y}{b^{u}+\left(A_{1}^{u}\right)^{n} X^{n}} \\
& \mathrm{LV}_{2}=\frac{q e^{\mu_{2}(t)}}{Y}\left(\mu_{2}^{\prime}(t)-a_{2}(t)-\frac{1}{T} \sum_{j=1}^{p} \ln \left(1+\xi_{2 k}\right)+\sigma_{2}^{2}(t)\right)+c\left(a_{2}(t)+\frac{1}{T} \sum_{j=1}^{p} \ln \left(1+\xi_{2 k}\right)\right) \\
& -c b_{2}(t) \frac{A_{2}(t) Y}{A_{1}(t) X}+q b_{2}(t) e^{\mu_{2}(t)} \frac{A_{2}(t)}{A_{1}(t) X}-\frac{c \sigma_{2}^{2}(t)}{2} \\
& \leq-\frac{q e^{\mu_{2}(t)}}{Y}\left(\left\langle a_{2}(t)-\sigma_{2}^{2}(t)\right\rangle_{T}+\frac{1}{T} \sum_{j=1}^{p} \ln \left(1+\xi_{2 k}\right)\right)+\frac{q b_{2}^{u} e^{\left|\mu_{2}^{u}\right|} A_{2}^{u}}{A_{1}^{l} X}-c b_{2}^{l} \frac{A_{2}^{l} Y}{A_{1}^{u} X} \\
& +c\left(a_{2}^{u}+\frac{1}{T} \sum_{j=1}^{p} \ln \left(1+\xi_{2 k}\right)-\frac{\left(\sigma_{2}^{2}\right)^{l}}{2}\right)
\end{aligned}
$$

Let $c=\left(a^{u} A_{1}^{u} A_{2}^{u} e^{\left|\mu_{1}^{u}\right|} / b^{l} b_{2}^{l} A_{2}^{l}\right)$, then we have

$\operatorname{LV}(t, X, Y) \leq \vartheta-b_{1}^{l} A_{1}^{l} X-\frac{A_{1}^{l} e^{\mu_{1}^{l}} \zeta-q e^{\left|\mu_{2}^{u}\right|} b_{2}^{u} A_{2}^{u}}{A_{1}^{l} X}-\frac{q e^{\mu_{2}^{l}}}{Y} \eta$

$$
-\frac{a^{l} A_{2}^{l} Y}{b_{1}^{u}+\left(A_{1}^{u}\right)^{n} X^{n}}
$$

where $\zeta$ and $\eta$ are defined as above, and

$$
\begin{aligned}
\vartheta:= & e^{\left|\mu_{1}^{u}\right|} b_{1}^{u} A_{1}^{u}+a_{1}^{u}+\frac{1}{T} \sum_{j=1}^{p} \ln \left(1+\xi_{1 k}\right)-\frac{\left(\sigma_{1}^{2}(t)\right)^{l}}{2} \\
& +\frac{a^{u} A_{1}^{u} A_{2}^{u} e^{\left|\mu_{1}^{u}\right|}}{b^{l} b_{2}^{l} A_{2}^{l}}\left(a_{2}^{u}+\frac{1}{T} \sum_{j=1}^{p} \ln \left(1+\xi_{2 k}\right)-\frac{\left(\sigma_{2}^{2}(t)\right)^{l}}{2}\right) .
\end{aligned}
$$

Take $q=\left(A_{1}^{l} e^{\mu_{1}^{l}} \zeta / 2 e^{\left|\mu_{2}^{u}\right|} b_{2}^{u} A_{2}^{u}\right)$, then 


$$
\begin{aligned}
\mathrm{LV}(t, X, Y) \leq & \vartheta-b_{1}^{l} A_{1}^{l} X-\frac{e^{\mu_{1}^{l}} \zeta}{2 X}-\frac{A_{1}^{l} e^{\mu_{1}^{l}+\mu_{2}^{l}-\left|\mu_{2}^{u}\right|}}{2 b_{2}^{u} A_{2}^{u} Y} \zeta \eta \\
& -\frac{a^{l} A_{2}^{l} Y}{b_{1}^{u}+\left(A_{1}^{u}\right)^{n} X^{n}}:=\Phi(X, Y) .
\end{aligned}
$$

Choose any small positive $\varepsilon_{1}$ and $\varepsilon_{2}$ such that

$$
\begin{aligned}
& 0<\varepsilon_{1}<\min \left\{\frac{b_{1}^{l} A_{1}^{l}}{\vartheta+1}, \frac{e^{\mu_{1}^{l}} \zeta}{2(\vartheta+1)}\right\}, \\
& 0<\varepsilon_{2}<\frac{A_{1}^{l} e^{\mu_{1}^{l}+\mu_{2}^{l}-\left|\mu_{2}^{u}\right|}}{2 b_{2}^{u} A_{2}^{u}(\vartheta+1)} \\
& \vartheta+1<\frac{a^{l} A_{2}^{l}\left(A_{1}^{u} \varepsilon_{1}\right)^{n}}{\varepsilon_{2}\left(b_{1}^{u}\left(A_{1}^{l} \varepsilon_{1}\right)^{n}+1\right)} .
\end{aligned}
$$

Define an open subset as follows:

$$
D_{\varepsilon_{1,2}}=\left\{(X, Y):(X, Y) \in\left(\varepsilon_{1}, \frac{1}{\varepsilon_{1}}\right) \times\left(\varepsilon_{2}, \frac{1}{\varepsilon_{2}}\right)\right\} .
$$

Obviously, $D_{\varepsilon_{1}}$ is compact and its component $D_{\varepsilon}^{C}=R_{+}^{2} / D_{\varepsilon}=\cup_{i=1}^{4} D_{\varepsilon}^{\varepsilon_{1}} D_{\varepsilon}^{i}$, where

$$
\begin{aligned}
& D_{\varepsilon}^{1}=\left\{(X, Y) \mid 0<X<\varepsilon_{1}\right\}, \\
& D_{\varepsilon}^{2}=\left\{(X, Y) \mid 0<Y<\varepsilon_{2}\right\}, \\
& D_{\varepsilon}^{3}=\left\{(X, Y) \mid X>\frac{1}{\varepsilon_{1}}\right\}, \\
& D_{\varepsilon}^{4}=\left\{(X, Y) \mid Y>\frac{1}{\varepsilon_{2}}\right\} .
\end{aligned}
$$

$$
x(t)=\frac{\prod_{0<t_{k}<t}\left(1+\xi_{1 k}\right) \exp \left(\int_{0}^{t}\left(a_{1}(s)-\left(\sigma_{1}^{2}(s) / 2\right)\right) \mathrm{d} s+\int_{0}^{t} \sigma_{1}(s) \mathrm{d} B(s)\right)}{(1 / x(0))+\int_{0}^{t} \prod_{0<t_{k}<s}\left(1+\xi_{1 k}\right) b_{1}(s) \exp \left(\int_{0}^{s}\left(a_{1}(\theta)-\left(\sigma_{1}^{2}(\theta) / 2\right)\right) \mathrm{d} \theta+\int_{0}^{s} \sigma_{1}(\theta) \mathrm{d} B(\theta)\right) \mathrm{d} s .}
$$

Remark 4. The existence of $T$-periodic solution can be obtained by Theorem 1 . The presentation of $x(t)$ and the global attractivity are referred to [21].

Lemma 6. For the solution $(x(t), y(t))$ of system (5), we have

$$
\begin{aligned}
& \limsup _{t \longrightarrow+\infty} P\left\{x(t)<\beta_{1}\right\}>1-\varepsilon, \\
& \underset{t \longrightarrow+\infty}{\limsup } P\left\{y(t)<\beta_{2}\right\}>1-\varepsilon,
\end{aligned}
$$

that is, the solution of (5) is stochastically ultimately bounded.

Proof. From the first equation of (5), we have
It is easy to verify that $\Phi(X, Y) \longrightarrow-\infty$ when $X \longrightarrow 0^{+}$, or $Y \longrightarrow 0^{+}$, or $X \longrightarrow \infty$, or $Y \longrightarrow \infty$. Therefore, $\operatorname{LV}(X, Y) \leq-1$ holds for any $(X, Y) \in R_{+}^{2} / D_{\varepsilon}$, which means the second condition of Lemma 1 holds. Using Lemma 1, then the existence of periodic solution of (10) is obtained. This completes the proof.

Remark 3. For system (5), if there is no impulsive effect, i.e., $\xi_{i k}=0(i=1,2)$, by Theorem 1 , we can obtain the sufficient conditions assuring the existence of $T$-periodic solution, which is in accordance with Theorem 3 in Reference [16]. And if $n=1$, i.e., the case of Holling type II functional response, Theorem 1 yields the same result as Theorem 3.3 in reference [23]. It is in this sense that we improved or generalized the main results in $[16,23]$.

\section{Extinction and Permanence in the Mean}

In this section, we discuss the extinction and permanence in the mean of system (5). Firstly, we provide a lemma on the presentation of the solution for an impulsive stochastic differential equation.

Lemma 5. For the following periodic impulsive differential equation

$$
\left\{\begin{array}{l}
\mathrm{d} x(t)=x(t)\left(a_{1}(t)-b_{1}(t) x(t)\right) \mathrm{d} t+\sigma_{1}(t) x(t) \mathrm{d} B(t), \quad t \neq t_{k}, \\
x\left(t_{k}^{+}\right)=\left(1+\xi_{1 k}\right) x\left(t_{k}\right),
\end{array}\right.
$$

let $x(t)$ be a solution with any given initial data $x(0)>0$. Then $\tilde{x}(t)$ is a unique positive T-periodic solution such that $\lim _{t \longrightarrow+\infty}|x(t)-\tilde{x}(t)|=0$, a.s, where

$$
\mathrm{d} x(t) \leq x(t)\left(a_{1}(t)-b_{1}(t) x(t)\right) \mathrm{d} t+\sigma_{1}(t) x(t) \mathrm{d} B_{1}(t) .
$$

Consider the following comparison system:

$$
\left\{\begin{array}{l}
\mathrm{d} u(t)=u(t)\left(a_{1}(t)-b_{1} u(t)\right) \mathrm{d} t+\sigma_{1}(t) u(t) \mathrm{d} B_{1}(t), \quad t \neq t_{k}, \\
u\left(t_{k}^{+}\right)=\left(1+\xi_{1 k}\right) u\left(t_{k}\right)
\end{array}\right.
$$

By Lemma 5, the solution $u(t)$ of (37) is positive and $T$-periodic, right continuous, and globally attractive. Therefore, $u(t)$ has maximum value and minimum value. Define $u^{*}=\max _{t \longrightarrow+\infty} u(t)$, then by comparison theorem for stochastic equation, for any sufficiently small positive $\varepsilon>0$, we have 


$$
x(t) \leq u(t) \leq u^{*}+\varepsilon:=\beta_{1} .
$$

Therefore, $\quad \limsup _{t \longrightarrow+\infty} P\left\{x(t)<\beta_{1}\right\}>1-\varepsilon \quad$ is established.

On the contrary, using $x(t) \leq \beta_{1}$, we can obtain from (5) that

$$
\mathrm{d} y(t) \leq y(t)\left(a_{2}(t)-b_{2}(t) \frac{y(t)}{\beta_{1}}\right) \mathrm{d} t+\sigma_{2}(t) y(t) \mathrm{d} B_{2}(t)
$$

In the same manner, we have

$$
y(t) \leq v(t) \leq v^{*}+\varepsilon:=\beta_{2},
$$

where $v(t)$ is the solution of the following stochastic comparison system:

$$
\left\{\begin{array}{l}
\mathrm{d} v(t)=v(t)\left(a_{2}(t)-b_{2}(t) \frac{y(t)}{\beta_{1}}\right) \mathrm{d} t+\sigma_{2}(t) v(t) \mathrm{d} B_{2}(t), \quad t \neq t_{k}, \\
v\left(t_{k}^{+}\right)=\left(1+\xi_{2 k}\right) v\left(t_{k}\right),
\end{array}\right.
$$

and $\quad v^{*}=\max _{t \rightarrow+\infty} v(t)$ Therefore, $\limsup _{t \longrightarrow+\infty} P\left\{y(t)<\beta_{2}\right\}>1-\varepsilon$. This completes the proof.

For convenience, denote

$$
\begin{aligned}
& \widehat{\zeta}:=\left\langle a_{1}(t)-\frac{\sigma_{1}^{2}(t)}{2}\right\rangle_{T}+\frac{1}{T} \sum_{j=1}^{p} \ln \left(1+\xi_{1 k}\right), \\
& \widehat{\eta}:=\left\langle a_{2}(t)-\frac{\sigma_{2}^{2}(t)}{2}\right\rangle_{T}+\frac{1}{T} \sum_{j=1}^{p} \ln \left(1+\xi_{2 k}\right), \\
& \widetilde{\zeta}:=\left\langle a_{1}(t)-\frac{\sigma_{1}^{2}(t)}{2}-\frac{a(t) A_{2}(t) \beta_{2}}{b(t)}\right\rangle_{T}+\frac{1}{T} \sum_{j=1}^{p} \ln \left(1+\xi_{1 k}\right) .
\end{aligned}
$$

(42)

Theorem 2. For system (5), the following results hold:

(i) If $\hat{\zeta}>0$, then $\langle x\rangle^{*} \leq M_{1}$, that is, species $x(t)$ is bounded in the mean. (ii) If $\hat{\eta}\rangle 0$, then $\langle y\rangle^{*} \leq M_{2}$ and $\langle y\rangle_{*} \geq m_{2}$, that is, species $y(t)$ is permanent in the mean.

(iii) If $\widehat{\zeta}<0$, then $\lim _{t \rightarrow+\infty} x(t)=0$, that is, species $x(t)$ is extinct, and if $\hat{\eta}<0$, then $\lim _{t \longrightarrow+\infty} y(t)=0$, that is, species $y(t)$ is extinct.

(iv) If $\widehat{\zeta}>0, \widehat{\eta}\left\langle 0\right.$, then $\langle x\rangle^{*} \leq M_{1}$ and $\langle x\rangle_{*} \geq \widetilde{m}_{1}$. If $\widetilde{\zeta}>0, \hat{\eta}\rangle 0$, then $\langle x\rangle^{*} \leq M_{1}$ and $\langle x\rangle_{*} \geq m_{1}$, that is, species $x(t)$ is permanent in the mean, where $\widetilde{m}_{1}, m_{i}, M_{i}(i=1,2)$ are constants defined later in the proof.

Proof. Make use of Lemma 3, we only need to prove these conclusions hold for (10) for some constants $\tilde{m}_{1}^{\prime}, m_{i}^{\prime}, M_{i}^{\prime}(i=1,2)$. For system $(10)$, by applying the Itô's formula to $\ln X$ and $\ln Y$, we have

$$
\begin{aligned}
& \mathrm{d} \ln X=\left(a_{1}(t)+\frac{1}{T} \sum_{j=1}^{p} \ln \left(1+\xi_{2 k}\right)-b_{1}(t) A_{1}(t) X-\frac{a(t) A_{2}(t) Y(t)}{b(t)+\left(A_{1}(t) X(t)\right)^{n}}-\frac{\sigma_{1}^{2}(t)}{2}\right) \mathrm{d} t+\sigma_{1}(t) \mathrm{d} B_{1}(t), \\
& \mathrm{d} \ln Y=\left(a_{2}(t)+\frac{1}{T} \sum_{j=1}^{p} \ln \left(1+\xi_{2 k}\right)-b_{2}(t) \frac{A_{2}(t) Y(t)}{A_{1}(t) X(t)}-\frac{\sigma_{2}^{2}(t)}{2}\right) \mathrm{d} t+\sigma_{2}(t) \mathrm{d} B_{2}(t)
\end{aligned}
$$

(i) If $\widehat{\zeta}>0$, then integrating both sides of (43) from 0 to $t$ yields

$$
\begin{aligned}
\ln \frac{X(t)}{X(0)} \leq & \frac{1}{t} \int_{0}^{t}\left(a_{1}(s)-\frac{\sigma_{1}^{2}(s)}{2}+\frac{1}{T} \sum_{j=1}^{p} \ln \left(1+\xi_{1 k}\right)\right) \mathrm{d} s \\
& -b_{1}^{u} A_{1}^{u} \int_{0}^{t} X(s) \mathrm{d} s+\frac{1}{t} \int_{0}^{t} \sigma_{1}(s) \mathrm{d} B_{1}(s) .
\end{aligned}
$$


Since $\lim _{t \longrightarrow+\infty}(1 / T) \int_{0}^{t} \sigma_{1}(s) \mathrm{d} B_{1}(s)=0$, then we obtain from Lemma 2 that

$$
\begin{aligned}
\langle X\rangle^{*} & \leq \frac{\left\langle a_{1}(t)-\left(\sigma_{1}^{2} / 2\right)\right\rangle_{T}+(1 / T) \sum_{j=1}^{p} \ln \left(1+\xi_{1 k}\right)}{b_{1}^{u} A_{1}^{u}} \\
& :=M_{1}^{\prime} .
\end{aligned}
$$

(ii) Lemma 4 implies the solution of (10) satisfies $X>0$, and hence there exists a positive constant $\varepsilon>0$ such that $x(t)>\varepsilon$. From (44), we have

$$
\begin{aligned}
\mathrm{d} \ln Y \geq & \left(a_{2}(t)-\frac{\sigma_{2}^{2}(t)}{2}+\frac{1}{T} \sum_{j=1}^{p} \ln \left(1+\xi_{2 k}\right)\right. \\
& \left.-\frac{b_{2}(t) A_{2}(t)}{A_{1}(t) \varepsilon} Y\right) \mathrm{d} t+\sigma_{2}(t) \mathrm{d} B_{2}(t),
\end{aligned}
$$

which leads to

$$
\begin{aligned}
\ln \frac{Y(t)}{Y(0)} \geq & \int_{0}^{t}\left(a_{2}(s)-\frac{\sigma_{2}^{2}(s)}{2}+\frac{1}{T} \sum_{j=1}^{p} \ln \left(1+\xi_{1 k}\right)\right) \mathrm{d} s \\
& -\frac{b_{2}^{u} A_{2}^{u}}{A_{1}^{l} \varepsilon} \int_{0}^{t} Y(s) \mathrm{d} s+\frac{1}{t} \int_{0}^{t} \sigma_{2}(s) \mathrm{d} B_{2}(s) .
\end{aligned}
$$

Under the condition $\widehat{\eta}>0$, we obtain from Lemma 2 that

$$
\begin{aligned}
\langle Y\rangle_{*} & \geq \frac{A_{1}^{l} \varepsilon\left(\left\langle a_{2}(t)-\left(\sigma_{2}^{2}(t) / 2\right)\right\rangle_{T}+(1 / T) \sum_{j=1}^{p} \ln \left(1+\xi_{1 k}\right)\right)}{b_{2}^{u} A_{2}^{u}} \\
& :=m_{2}^{\prime} .
\end{aligned}
$$

On the contrary, combining Lemma 6, we have

$$
\begin{aligned}
\mathrm{d} \ln Y \leq & \left(a_{2}(t)-\frac{\sigma_{2}^{2}(t)}{2}+\frac{1}{T} \sum_{j=1}^{p} \ln \left(1+\xi_{1 k}\right)\right. \\
& \left.-\frac{b_{2}^{l} A_{2}^{l}}{A_{1}^{u} \beta_{1}} Y\right) \mathrm{d} t+\sigma_{2}(t) \mathrm{d} B_{2}(t) .
\end{aligned}
$$

Integrating both sides of above inequality yields

$$
\begin{aligned}
\ln \frac{Y(t)}{Y(0)} \leq & \frac{1}{t} \int_{0}^{t}\left(a_{2}(s)-\frac{\sigma_{2}^{2}(s)}{2}+\frac{1}{T} \sum_{j=1}^{p} \ln \left(1+\xi_{1 k}\right)\right) \mathrm{d} s \\
& -\frac{b_{2}^{l} A_{2}^{l}}{A_{1}^{u} \beta_{1}} \int_{0}^{t} Y(s) \mathrm{d} s+\frac{1}{t} \int_{0}^{t} \sigma_{2}(t) \mathrm{d} B_{2}(t) .
\end{aligned}
$$

If $\hat{\eta}>0$, using Lemma 2 again, then

$$
\begin{aligned}
\langle Y\rangle^{*} & \leq \frac{A_{1}^{u} \beta_{1}\left(\left\langle a_{2}(t)-\left(\sigma_{2}^{2}(t) / 2\right)\right\rangle_{T}+(1 / T) \sum_{j=1}^{p} \ln \left(1+\xi_{1 k}\right)\right)}{b_{2}^{l} A_{2}^{l}} \\
& :=M_{2}^{\prime} .
\end{aligned}
$$

Therefore, species $Y(t)$ is permanent in the mean.

(iii) If $\widehat{\zeta}<0$, by Case (i), obviously $\lim _{t \longrightarrow+\infty} X(t)=0$. If $\widehat{\eta}<0$, similarly, by the proof of Case (ii), we have $\lim _{t \rightarrow+\infty} Y(t)=0$.

(iv) If $\widehat{\zeta}>0$, then Case (i) indicates $\langle X\rangle^{*} \leq M_{1}^{\prime}$. If $\hat{\eta}<0$, by use of Case (ii), we have $\lim _{t \longrightarrow+\infty} Y(t)=0$, then (43) reads

$$
\mathrm{d} \ln X=\left(a_{1}(t)+\frac{1}{T} \sum_{j=1}^{p} \ln \left(1+\xi_{1 k}\right)-b_{1}(t) A_{1}(t) X-\frac{\sigma_{1}^{2}(t)}{2}\right) \mathrm{d} t+\sigma_{1}(t) \mathrm{d} B_{1}(t)
$$

and hence,

$$
\begin{aligned}
\mathrm{d} \ln X \geq & \left(a_{1}(t)+\frac{1}{T} \sum_{j=1}^{p} \ln \left(1+\xi_{1 k}\right)\right. \\
& \left.-b_{1}^{u} A_{1}^{u} X-\frac{\sigma_{1}^{2}(t)}{2}\right) \mathrm{d} t+\sigma_{1}(t) \mathrm{d} B_{1}(t) .
\end{aligned}
$$

According to Lemma 2, then

$$
\langle X\rangle_{*} \geq \frac{\left\langle a_{1}(t)-\left(\sigma_{1}^{2}(t) / 2\right)\right\rangle_{T}+(1 / T) \sum_{j=1}^{p} \ln \left(1+\xi_{1 k}\right)}{b_{1}^{u} A_{1}^{u}}:=\widetilde{m}_{1}^{\prime} .
$$

If $\widetilde{\zeta}>0$, then obviously $\widehat{\zeta}>0$, and hence $\langle X\rangle^{*} \leq M_{1}^{\prime}$. Therefore, 


$$
\widetilde{m}_{1}^{\prime} \leq\langle X\rangle \leq M_{1}^{\prime}
$$

Furthermore, if $\hat{\eta}>0$, then from (33) and Lemma 6, we have

$$
d \ln X \geq\left(a_{1}(t)+\frac{1}{T} \sum_{j=1}^{p} \ln \left(1+\xi_{1 k}\right)-b_{1}^{u} A_{1}^{u} X-\frac{a(t) A_{2}(t) \beta_{2}}{b(t)}-\frac{\sigma_{1}^{2}(t)}{2}\right) \mathrm{d} t+\sigma_{1}(t) \mathrm{d} B_{1}(t)
$$

and hence,

$$
\ln \frac{X(t)}{X(0)} \geq \frac{1}{t} \int_{0}^{t}\left(a_{1}(s)-\frac{\sigma_{1}^{2}(s)}{2}+\frac{1}{T} \sum_{j=1}^{p} \ln \left(1+\xi_{1 k}\right)-\frac{a(s) A_{2}(s) \beta_{2}}{b(s)}\right) \mathrm{d} s-b_{1}^{u} A_{1}^{u} \int_{0}^{t} X(s) \mathrm{d} s+\frac{1}{t} \int_{0}^{t} \sigma_{1}(t) \mathrm{d} B_{1}(t)
$$

Then

$$
\langle X\rangle_{*} \geq \frac{\left\langle a_{1}(t)-\left(\sigma_{1}^{2}(t) / 2\right)-\left(a(t) A_{2}(t) \beta_{2} / b(t)\right)\right\rangle_{T}+(1 / T) \sum_{j=1}^{p} \ln \left(1+\xi_{1 k}\right)}{b_{1}^{u} A_{1}^{u}:=m_{1}^{\prime} .}
$$

Combining $\langle X\rangle^{*} \leq M_{1}^{\prime}$, then species $X(t)$ is permanent in the mean.

To summarize, the above conclusions hold for system (10). Using Lemma 3, the required assertion is directly obtained. The proof is completed.

Remark 5. For system (5), if the impulsive is absent, then Theorem 2 implies the sufficient conditions of the extinction of species $x(t)$ or $y(t)$, which is accordance with Theorem 2 in [16].

\section{Stochastic Permanence}

In this section, we consider the stochastic permanence of (5).

Theorem 3. If $\widehat{\zeta}>0, \widehat{\eta}>0$, then system (5) is stochastically permanent.

Proof. This proof is motivated by Reference [21]. Let $\Omega_{t}^{\varepsilon}=\left\{\omega \in \Omega, X(t)<\beta_{1}, Y(t)<\beta_{2}\right\}, \quad \Omega_{\infty}^{\varepsilon}=\{\omega \in \Omega$, $\left.\limsup _{t \rightarrow+\infty} P\left\{\Omega_{t}^{\varepsilon}\right\}>1-\varepsilon\right\}$, then Lemma 6 implies that $P\left\{\Omega_{\infty}^{\varepsilon}\right\}>1-\varepsilon$. Hence, for any $\omega \in \Omega_{\infty}^{\varepsilon}$, there exists $k_{1}>0$ such that $X(t)<\beta_{1}, Y(t)<\beta_{2}$ for any $t \geq k_{1} T$.

On the contrary, by the assumption $\widehat{\zeta}>0$, we can choose a positive constant $\varepsilon>0$ such that

$$
\widehat{\zeta}-b_{1}^{u} A_{1}^{u} \varepsilon-\frac{a^{u} A_{1}^{u} A_{2}^{u} \varepsilon}{b^{l} b_{2}^{l} A_{2}^{l}}>0
$$

Firstly, we prove that there exists $\varsigma_{1}>0$, for any $\omega \in \Omega_{\infty}^{\varepsilon}$, we have

$$
\liminf _{t \longrightarrow+\infty} P\left\{X(t)>\varsigma_{1}\right\}>1-\varepsilon .
$$

Suppose it is not true, i.e., there exist a solution $(X, Y)$ with initial data $X(0)>0, Y(0)>0$, a positive integer $k_{2}>k_{1}$, and a nonempty set $\Omega_{1} \subset \Omega_{t}^{\varepsilon}$ with $P\left(\Omega_{1}\right)>0$ such that $X(t, \omega)<\varsigma_{1}$ for any $\omega \in \Omega_{1}$ and $t \geq k_{2} T$. From (44), we have

$$
\begin{aligned}
\mathrm{d} \ln Y= & \left(a_{2}(t)+\frac{1}{T} \sum_{j=1}^{p} \ln \left(1+\xi_{2 k}\right)-b_{2}(t) \frac{A_{2}(t) Y(t)}{A_{1}(t) X(t)}-\frac{\sigma_{2}^{2}(t)}{2}\right) \mathrm{d} t+\sigma_{2}(t) \mathrm{d} B_{2}(t) \\
& \leq\left(a_{2}(t)-\frac{\sigma_{2}^{2}(t)}{2}+\frac{1}{T} \sum_{j=1}^{p} \ln \left(1+\xi_{2 k}\right)\right)-b_{2}^{l} \frac{A_{2}^{l} Y(t)}{A_{1}^{u} \varsigma_{1}}+\sigma_{2}(t) \mathrm{d} B_{2}(t) .
\end{aligned}
$$


Then Lemma 2 implies

On the contrary,

$\langle Y\rangle^{*} \leq \frac{A_{1}^{u}\left(\left\langle a_{2}(t)-\left(\sigma_{2}^{2} / 2\right)\right\rangle+(1 / T) \sum_{j=1}^{p} \ln \left(1+\xi_{2 k}\right)\right) \varsigma_{1}}{b_{2}^{l} A_{2}^{l}}:=\kappa$

$$
\mathrm{d} \ln X=\left(a_{1}(t)+\frac{1}{T} \sum_{j=1}^{p} \ln \left(1+\xi_{2 k}\right)-2 b_{1}(t) A_{1}(t) X-\frac{a(t) A_{2}(t) Y(t)}{b(t)+\left(A_{1}(t) X(t)\right)^{n}}-\frac{\sigma_{1}^{2}(t)}{2}\right) \mathrm{d} t+\sigma_{1}(t) \mathrm{d} B_{1}(t)
$$

Integrating (64) from $k_{2} T$ to $t$ yields

$$
\begin{aligned}
\ln X(t)= & \ln X\left(k_{2} T\right)+\int_{k_{2} T}^{t}\left(a_{1}(s)+\frac{1}{T} \sum_{j=1}^{p} \ln \left(1+\xi_{2 k}\right)-\frac{\sigma_{1}^{2}(s)}{2}\right) \mathrm{d} s-\int_{k_{2} T}^{t} 2 b_{1}(s) A_{1}(s) X \mathrm{~d} s \\
& -\int_{k_{2} T}^{t} \frac{a(s) A_{2}(s) Y(s)}{b(s)+\left(A_{1}(s) X(s)\right)^{n}} \mathrm{~d} s+\int_{k_{2} T}^{t} \sigma_{1}(s) \mathrm{d} B_{1}(s) .
\end{aligned}
$$

Then we derive from (65) that

$$
\begin{aligned}
\liminf _{t \longrightarrow+\infty} \frac{\ln X}{t} \geq \liminf _{t \longrightarrow+\infty}\left\langle a_{1}(t)-\frac{\sigma_{1}^{2}(t)}{2}\right\rangle_{T}+\frac{1}{T} \sum_{j=1}^{p} \ln \left(1+\xi_{1 k}\right) \\
-b_{1}^{u} A_{1}^{u} \varsigma_{1}-\frac{a^{u} A_{2}^{u} \kappa}{b^{l}}>0 .
\end{aligned}
$$

Then we conclude $X(t) \longrightarrow+\infty$ as $t \longrightarrow+\infty$, which contradicts with the boudedness of $X(t)$. Hence we have $\lim \inf _{t \longrightarrow+\infty} P\left\{X(t)>\varsigma_{1}\right\}>1-\varepsilon$ for any $\omega \in \Omega_{\infty}^{\varepsilon}$.
Secondly, we prove there exists a constant $\alpha_{1}(\varepsilon)>0$ such that

$$
\liminf _{t \longrightarrow+\infty} P\left\{\omega \in \Omega_{\infty}^{\varepsilon}: \alpha_{1}(\varepsilon) \leq X(t, \omega) \leq \beta_{1}(\varepsilon)\right\} \geq 1-\varepsilon .
$$

We claim that $\liminf _{t \longrightarrow+\infty} P\left\{x(t)>\alpha_{1}\right\}>1-\varepsilon$ holds for all $\omega \in \Omega_{\infty}^{\varepsilon}$. If not, then there exists a sequence $\left\{v_{n}\right\} \subset R_{+}$and a nonempty set $\Omega_{2} \subset \Omega_{t}^{\varepsilon}$ with $P\left(\Omega_{2}\right)>0$, and a positive integer $k_{3}\left(k_{3}>k_{1}\right)$ such that $\sup _{t>k_{3} T} X\left(t, v_{n}, \omega\right)$ $<\left(\alpha_{1}(\varepsilon) / n+1\right)$ for all $\omega \in \Omega_{2}$, where $\left(X\left(t, v_{n}\right), Y\left(t, v_{n}\right)\right)$ is the solution of (10) with initial data $\nu_{n}$. Using (64), for all $t \geq k_{3} T$, we have

$$
\frac{\mathrm{d}\left[\mathbb{E} \ln X\left(t, v_{n}\right) I_{\Omega_{2}}\right]}{\mathrm{d} t} \geq\left[\left(a_{1}(t)+\frac{1}{T} \sum_{j=1}^{p} \ln \left(1+\xi_{1 k}\right)-\frac{\sigma_{1}^{2}(t)}{2}\right)-2 b_{1}(t) A_{1}(t) \beta_{1}(\varepsilon)-\frac{a(t) A_{2}(t) \beta_{2}(\varepsilon)}{b(t)}\right] P\left(\Omega_{2}\right)
$$

According to the previous proof, there is a nonempty set $\Omega_{3} \subseteq \Omega_{2}$ where $P\left(\Omega_{3}\right)>0$, and two positive integers $k_{4}$ and $k_{5}$ with $k_{5}>k_{4}>k_{3}$ such that

$$
\begin{aligned}
& X\left(k_{4} T, v_{n}\right) \geq \alpha_{1}(\varepsilon), \\
& X\left(k_{5} T, v_{n}\right) \leq \frac{\alpha_{1}(\varepsilon)}{n+1}
\end{aligned}
$$

hold for all $\omega \in \Omega_{3}$. Define

$$
\begin{aligned}
& \tau^{*}=\inf \left\{t: X\left(t, v_{n}\right)>\frac{\alpha_{1}(\varepsilon)}{n+1}, \omega \in \Omega_{3}, t>k_{5} T\right\}, \\
& \tau_{*}=\sup \left\{t<\tau^{*}: X\left(t, v_{n}\right)<\alpha_{1}(\varepsilon), \omega \in \Omega_{3}, t>k_{4} T\right\} .
\end{aligned}
$$

Then $X\left(\tau^{*}, v_{n}\right)=\left(\alpha_{1}(\varepsilon) / n+1\right), X\left(\tau_{*}, v_{n}\right)=\alpha_{1}(\varepsilon)$, and for any $t \in\left(\tau_{*}, \tau^{*}\right)$ and $\omega \in \Omega_{3}$, we have

$$
\frac{\alpha_{1}(\varepsilon)}{n+1}<X\left(t, v_{n}\right)<\alpha_{1}(\varepsilon) \text {. }
$$

Integrating both sides of (65) from $\tau_{*}$ to $\tau^{*}$ yields 


$$
\begin{aligned}
& \int_{\tau_{*}}^{\tau^{*}}\left[\frac{\sigma_{1}^{2}(t)}{2}-a_{1}(t)-\frac{1}{T} \sum_{j=1}^{p} \ln \left(1+\xi_{1 k}\right)+2 b_{1}(t) A(t) \beta_{1}(\varepsilon)+\frac{a(t) B(t) \beta_{2}(\varepsilon)}{b(t)}\right] \mathrm{d} t \\
& \geq-\mathbb{E}\left[\ln X\left(\tau^{*}, v_{n}\right) I_{\Omega_{3}}\right]+\mathbb{E}\left[\ln X\left(\tau_{*}, v_{n}\right) I_{\Omega_{3}}\right] \\
& =\ln (n+1) P\left(\Omega_{3}\right),
\end{aligned}
$$

which leads to $\tau^{*}-\tau_{*} \longrightarrow+\infty$ as $n \longrightarrow+\infty$.

On the contrary, by the given condition, there exist two constants $\tau_{0}$ and $\gamma$ such that for all $t \geq \tau_{0}$ and $l>0$, we have

$$
\eta_{1}(l+t)-\int_{l}^{l+t}\left(b_{1}^{u} A_{1}^{u} \varepsilon+\frac{a^{u} A_{1}^{u} A_{2}^{u} \varepsilon}{b^{l} b_{2}^{l} A_{2}^{l}}\right) \mathrm{d} s>\gamma, \quad \text { a.s. }
$$

Proof. where $\quad \eta_{1}(t)=(1 / T) \int_{0}^{t}\left(a_{1}(s) \quad-\left(\sigma_{1}^{2}(s) / 2\right)\right) \mathrm{d} s$ $+(1 / T) \sum_{j=1}^{p} \ln \left(1+\xi_{2 k}\right)$.

For any $t \in\left[\tau_{*}, \tau^{*}\right]$ and $\omega \in \Omega_{3}$, we have

$$
\begin{aligned}
\mathrm{d} \ln Y \leq & \left(a_{2}(t)-\frac{\sigma_{2}^{2}(t)}{2}+\frac{1}{T} \sum_{j=1}^{p} \ln \left(1+\xi_{2 k}\right)\right)-\frac{b_{2}^{l} A_{2}^{l} Y(t)}{A_{1}^{u} \alpha_{1}} \\
& +\sigma_{2}(t) \mathrm{d} B_{2}(t) .
\end{aligned}
$$

The previous proof shows that there exists a positive integer $k_{6}>t_{0} / T$ such that $\langle Y\rangle^{*} \leq \kappa$ for any $t>k_{6} T+\tau_{*}$ and $\omega \in \Omega_{3}$. Due to $T^{*}-T_{*} \longrightarrow \infty$, then there exists a positive integer $n_{0}>0$ such that $\tau^{*}>k_{6} T+\tau^{*}$ for any $n \geq n_{0}$, and hence

$$
\langle Y\rangle^{*} \leq \frac{A_{1}^{u}\left(\left\langle a_{2}(t)-\left(\sigma_{2}^{2}(t) / 2\right)\right\rangle_{T}+(1 / T) \sum_{j=1}^{p} \ln \left(1+\xi_{2 k}\right)\right) \alpha_{1}}{b_{2}^{l} A_{2}^{l}}
$$
$:=\widetilde{\kappa}$,

for any $t \in\left[k_{6} T+\tau_{*}, \tau^{*}\right]$ and $\omega \in \Omega_{3}$. Using (65) again, we have

$$
\left.\mathbb{E}\left[\ln \left(X\left(t_{n}, v_{n}\right)\right) I_{\Omega_{3}}\right] \geq \mathbb{E}\left[\ln X\left(k_{6} T+\tau_{*}\right), v_{n} * * * I_{\Omega_{3}}\right]+\mathbb{E}\left[\eta_{1}\left(\tau^{*}\right)-\int_{k_{6} T+\tau_{*}}^{\tau^{*}}\left(b_{1}^{u} A_{1}^{u} \alpha_{1}+\widetilde{\kappa}\right) \mathrm{d} s\right) I_{\Omega_{3}}\right]
$$

which leads to

$$
\left.\frac{\alpha_{1}(\varepsilon)}{n+1} \geq \frac{\alpha_{1}(\varepsilon)}{n+1} \exp \left\{\frac{1}{P\left(\Omega_{3}\right)} \mathbb{E}\left[\eta_{1}\left(\tau^{*}\right)-\int_{k_{6} T+\tau_{*}}^{\tau^{*}}\left(b_{1}^{u} A_{1}^{u} \alpha_{1}+\widetilde{\kappa}\right) \mathrm{d} s\right) I_{\Omega_{3}}\right]\right\} \geq \frac{\alpha_{1}(\varepsilon)}{n+1} e^{\gamma}
$$

Obviously it implies a contradiction, and our claim is obtained. Therefore

$\liminf _{t \longrightarrow+\infty} P\left\{\omega \in \Omega_{\infty}^{\varepsilon}: \alpha_{1}(\varepsilon) \leq X(t, \omega) \leq \beta_{1}(\varepsilon)\right\} \geq 1-\varepsilon$ holds.

In a similar way, we can derive that $\liminf _{t \longrightarrow+\infty} P\left\{\omega \in \Omega_{\infty}^{\varepsilon}: \alpha_{2}(\varepsilon) \leq Y(t, \omega) \leq \beta_{2}(\varepsilon)\right\} \geq 1-\varepsilon$.
Finally, making use of Lemma 3 yielding the required assertion. The proof is completed.

\section{Examples and Simulations}

In this section, we give some examples and apply the Melstein method [31] to illustrate our theoretical results and reveal the effects of random disturbance and impulsive 


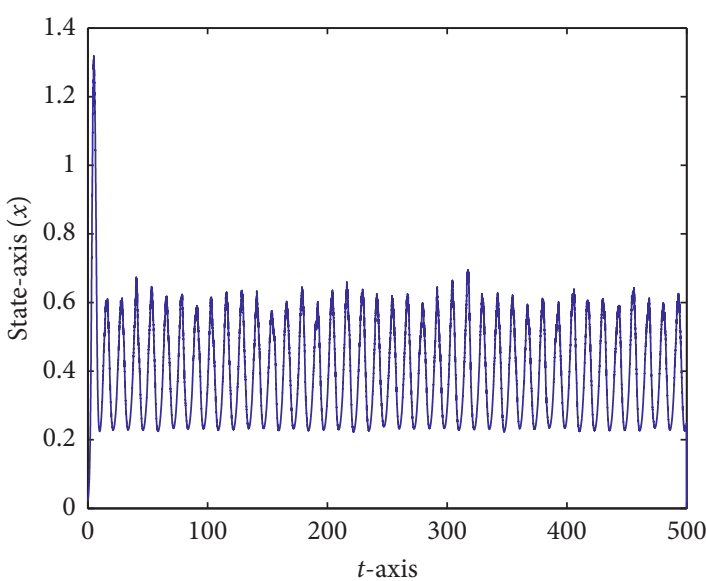

$-x(\mathrm{t})$

(a)

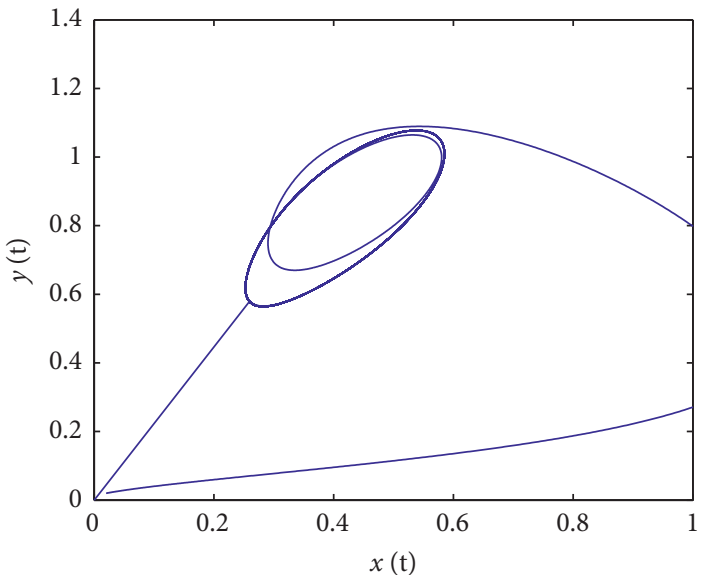

— Deterministic case

(c)

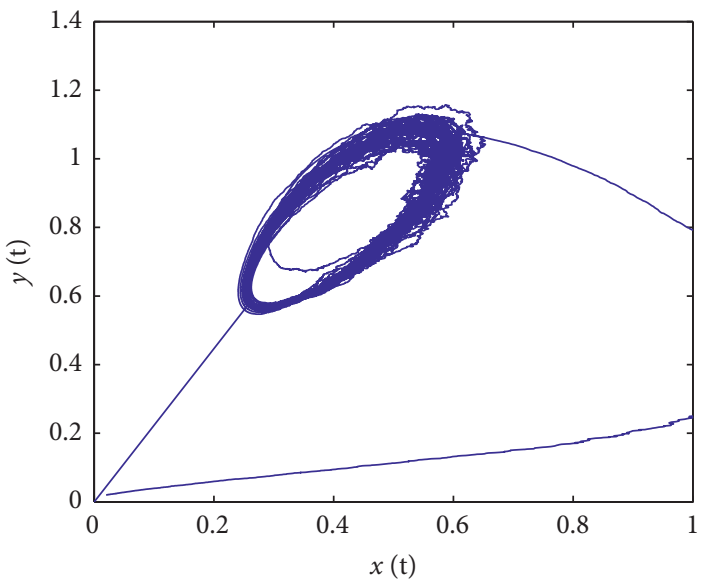

- Stochastic case

(e)

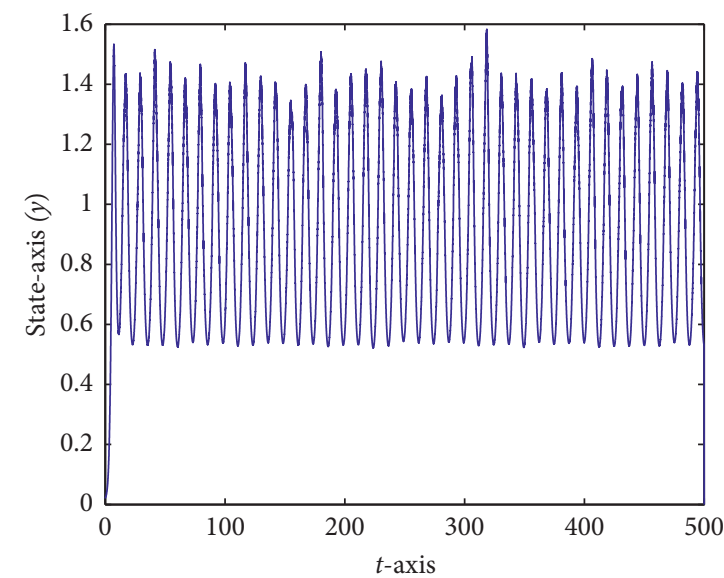

$-y(\mathrm{t})$

(b)

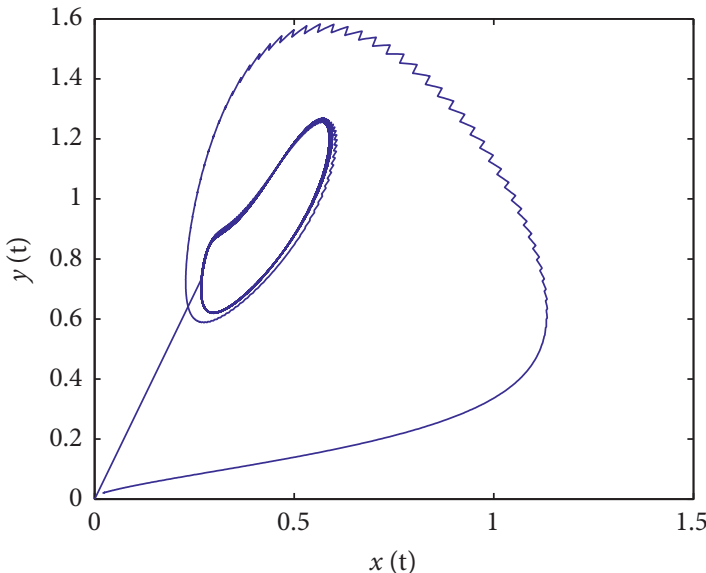

_- Impulsive case

(d)

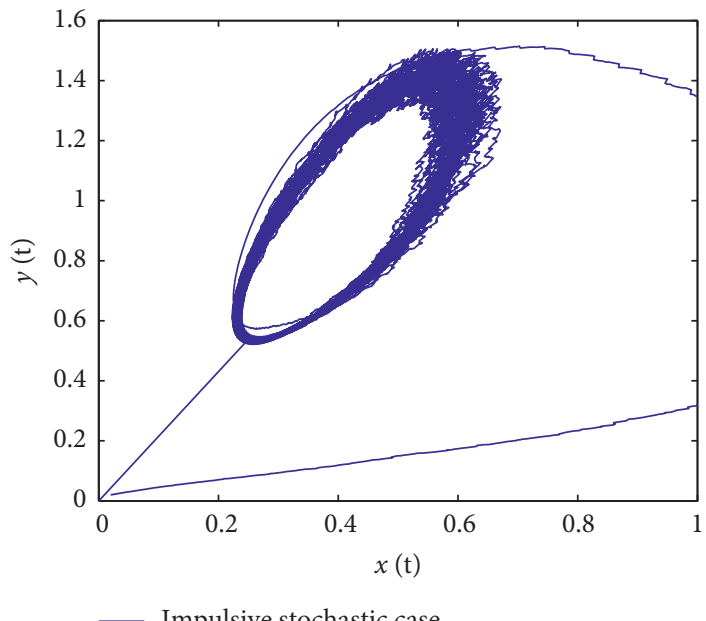

(f)

Figure 1: Periodic Markov process of (5) with $n=1$ and $x(0)=y(0)=0.02$. (a) The time series graph of $x$. (b) The time series graph of $y$. (c) The phase graph of deterministic system $\left(\xi_{i k}=0, \sigma_{i}(t)=0, i=1,2\right)$. (d) The phase graph of impulsive system $\left(\sigma_{i}(t)=0, i=1,2\right)$. (e) The phase graph of stochastic system $\left(\sigma_{i}(t)=0, i=1,2\right)$. (f) The phase graph of stochastic impulsive system with $\sigma_{1}(t)=0.02+0.02 \sin (t / 2), \sigma_{2}(t)=0.01+0.01 \sin (t / 2), \xi_{1 k}=0.01+0.01 \sin (t / 2), \xi_{2 k}=0.01+0.01 \sin (t / 2), t_{k}=0.1 k, k \in N$. 


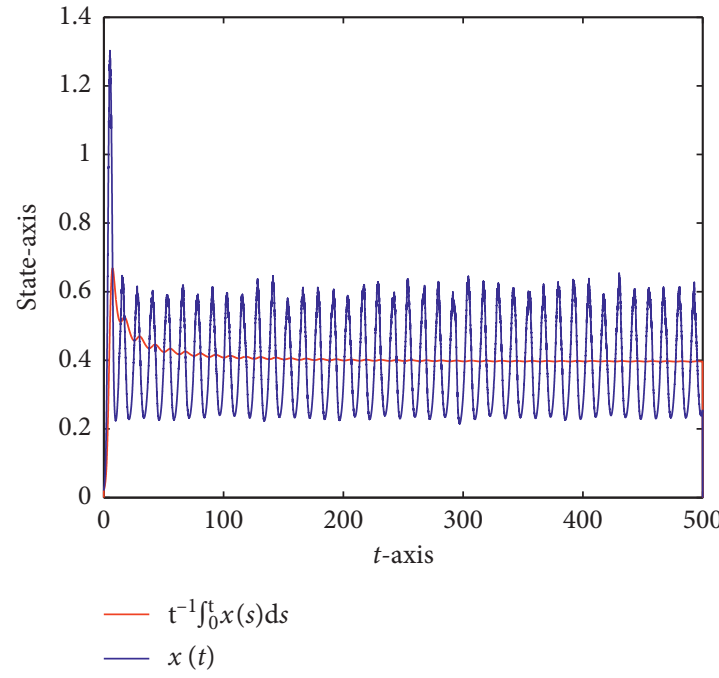

(a)

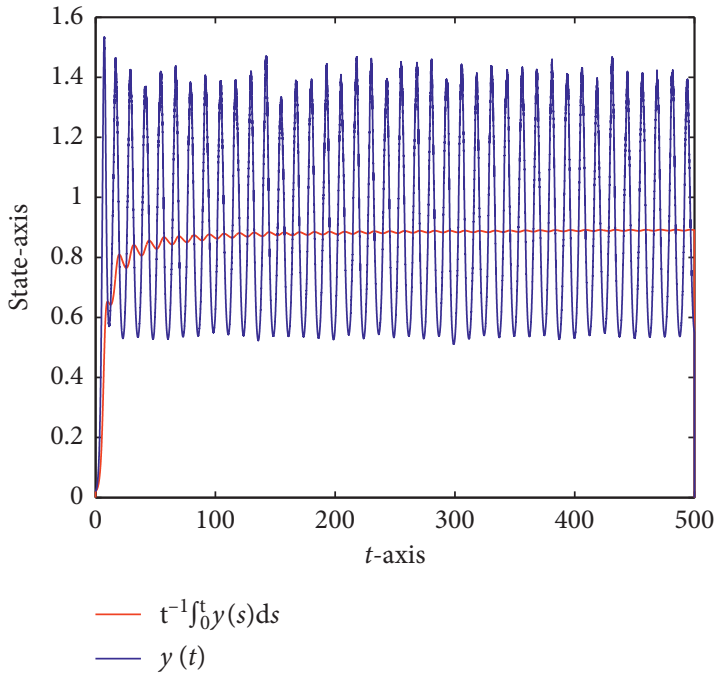

(b)

Figure 2: Permanence in the mean of (5) with $n=1$ and $x(0)=y(0)=0.02$. (a) The permanence in the mean of $x$. (b) The permanence in the mean of $y$.

factors. For system (5), except some special mentions later, we always take

$$
\begin{aligned}
a_{1}(t) & =1+0.2 \sin \left(\frac{t}{2}\right) \\
b(t) & =0.8+0.5 \cos \left(\frac{t}{2}\right) \\
\sigma_{1}(t) & =0.02+0.02 \sin \left(\frac{t}{2}\right) \\
\xi_{2 k} & =0.01+0.01 \sin \left(\frac{t}{2}\right) \\
b_{1}(t) & =0.8+0.1 \cos \left(\frac{t}{2}\right) \\
a_{2}(t) & =0.6+0.1 \sin \left(\frac{t}{2}\right) \\
\sigma_{2}(t) & =0.01+0.01 \sin \left(\frac{t}{2}\right) \\
t_{k} & =0.1 k T, \quad k \in N, p=10 . \\
\xi_{1 k} & =0.01+0.01 \sin \left(\frac{t}{2}\right) . \\
a(t) & =1+0.3 \cos \left(\frac{t}{2}\right) \\
b_{2}(t) & =0.3+0.05 \sin \left(\frac{t}{2}\right) \\
& =0
\end{aligned}
$$

For system (5), if $n=1$, then we get the stochastic Holling-Tanner system. By computation,

$$
\begin{gathered}
\zeta:=\left\langle a_{1}(t)-\sigma_{1}^{2}(t)\right\rangle_{T}+\frac{1}{T} \sum_{j=1}^{p} \ln \left(1+\xi_{1 k}\right)=0.9994>0, \\
\eta:=\left\langle a_{2}(t)-\sigma_{2}^{2}(t)\right\rangle_{T}+\frac{1}{T} \sum_{j=1}^{p} \ln \left(1+\xi_{2 k}\right)=0.5998>0 .
\end{gathered}
$$

Then condition $\left(H_{1}\right)$ holds, and Theorem 1 implies that the solution of the stochastic Holling-Tanner system is a $4 \pi$-periodic Markovian process (Figure 1). Figures 1(a) and 1(b) are the time series graphs of $x$ and $y$, respectively. Under the condition $\left(H_{1}\right)$ holding, Figures $1(\mathrm{c})-1(\mathrm{f})$ are the phase graphs of periodic solution of deterministic system $\left(\sigma_{i}(t)=0, \xi_{i k}=0, i=1,2\right)$, impulsive system $\left(\sigma_{i}(t)=0, \xi_{i k} \neq\right.$ $0, i=1,2)$, stochastic system $\left(\sigma_{i}(t) \neq 0, \xi_{i k}=0, i=1,2\right)$, and stochastic impulsive system $\left(\sigma_{i}(t) \neq 0, \xi_{i k} \neq 0, i=1,2\right)$, respectively. Similarly, the conditions of Case (iv) of Theorems 2 and 3 hold, and the system is permanent in the mean and stochastically permanent, see Figures 2(a), 2(b), and 3, respectively. On the contrary, let all parameters are as before except $\sigma_{1}(t)=1.5+0.02 \sin (t / 2), \sigma_{2}(t)=1.2+0.01$ $\sin (t / 2) \quad$ or $\xi_{1 k}=-0.1+0.01 \sin \left(t_{k} / 2\right), \xi_{2 k}=-0.1+0.01$ $\sin \left(t_{k} / 2\right), t_{k}=0.1 k, k \in N$, then an easy computing yields $\widehat{\zeta}<0, \widehat{\eta}<0$; therefore, $x$ and $y$ are both extinct, illustrated in Figures 4(a) and 4(b), respectively. If $\sigma_{1}(t)=1+0.02$ $\sin (t / 2), \sigma_{2}(t)=0.01+0.01 \sin (t / 2)$, then Theorem 2 indicates $y$ is always permanent (Figure 5(a)). If $\sigma_{1}(t)=0.02+$ $0.02 \sin (t / 2), \sigma_{2}(t)=1+0.01 \sin (t / 2)$, then Theorem 2 implies that $x$ is permanent, but $y$ is extinct (Figure 5(b)). For system (5), if $n=2$, then we get a stochastic periodic Holling type IV predator-prey system. It is clear that Theorem 1 implies that the solution is a periodic Markovian process (Figure 6). Figures $6(\mathrm{a})-6(\mathrm{~d})$ are the phase graphs of periodic solution of deterministic system $\left(\sigma_{i}(t)=0, \xi_{i k}=0, i=1,2\right)$, impulsive 


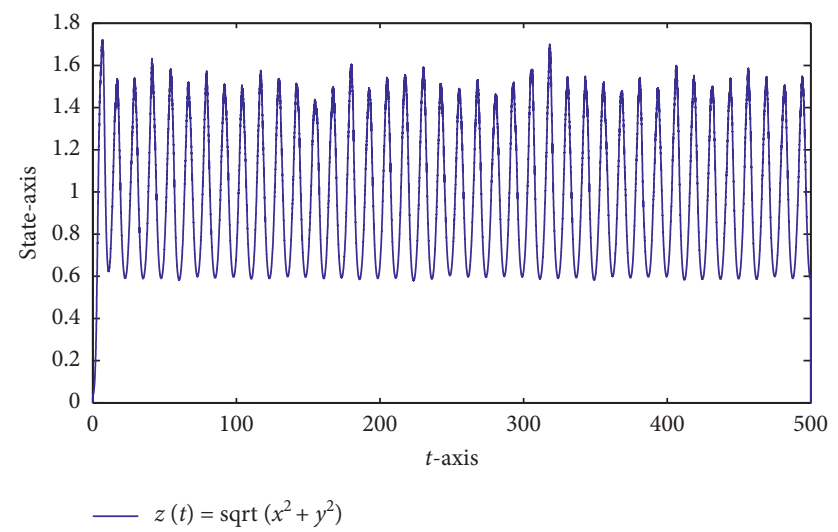

FIGURE 3: Stochastic persistence of (5) with $n=1$ and $x(0)=y(0)=0.02$.
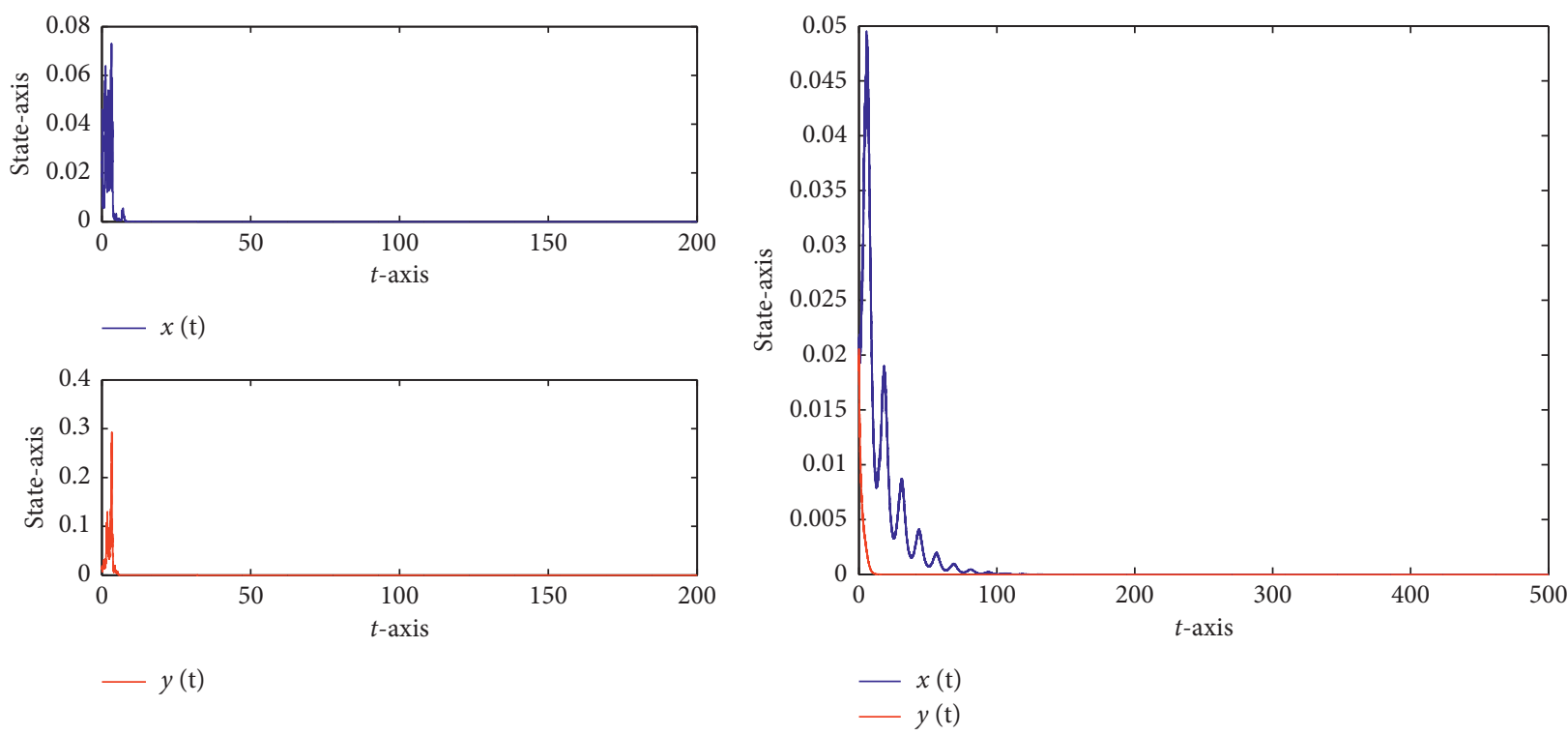

(a)

(b)

Figure 4: The extinction of all species of system (5) with $n=1$ and $x(0)=y(0)=0.02$. (a) The extinction of $x$ and $y$ with $\sigma_{1}(t)=1.5+0.02 \sin (t / 2), \sigma_{2}(t)=1.2+0.01 \sin (t / 2)$. (b) The extinction of $x$ and $y$ with $\xi_{1 k}=-0.1+0.01 \quad \sin \left(t_{k} / 2\right), \xi_{2 k}=-0.1$ $+0.01 \sin \left(t_{k} / 2\right), t_{k}=0.1 k, k \in N$.

system $\quad\left(\sigma_{i}(t)=0, \xi_{i k} \neq 0, i=1,2\right), \quad$ stochastic $\quad$ system $\left(\sigma_{i}(t) \neq 0, \xi_{i k}=0, i=1,2\right)$, and stochastic impulsive system $\left(\sigma_{i}(t) \neq 0, \xi_{i k} \neq 0, i=1,2\right)$, respectively. Similarly, the conditions of Case (iv) of Theorems 2 and 3 hold, and the system is permanent in the mean and stochastically permanent, see Figures $7(\mathrm{a}), 7(\mathrm{~b})$, and 8 , respectively. 

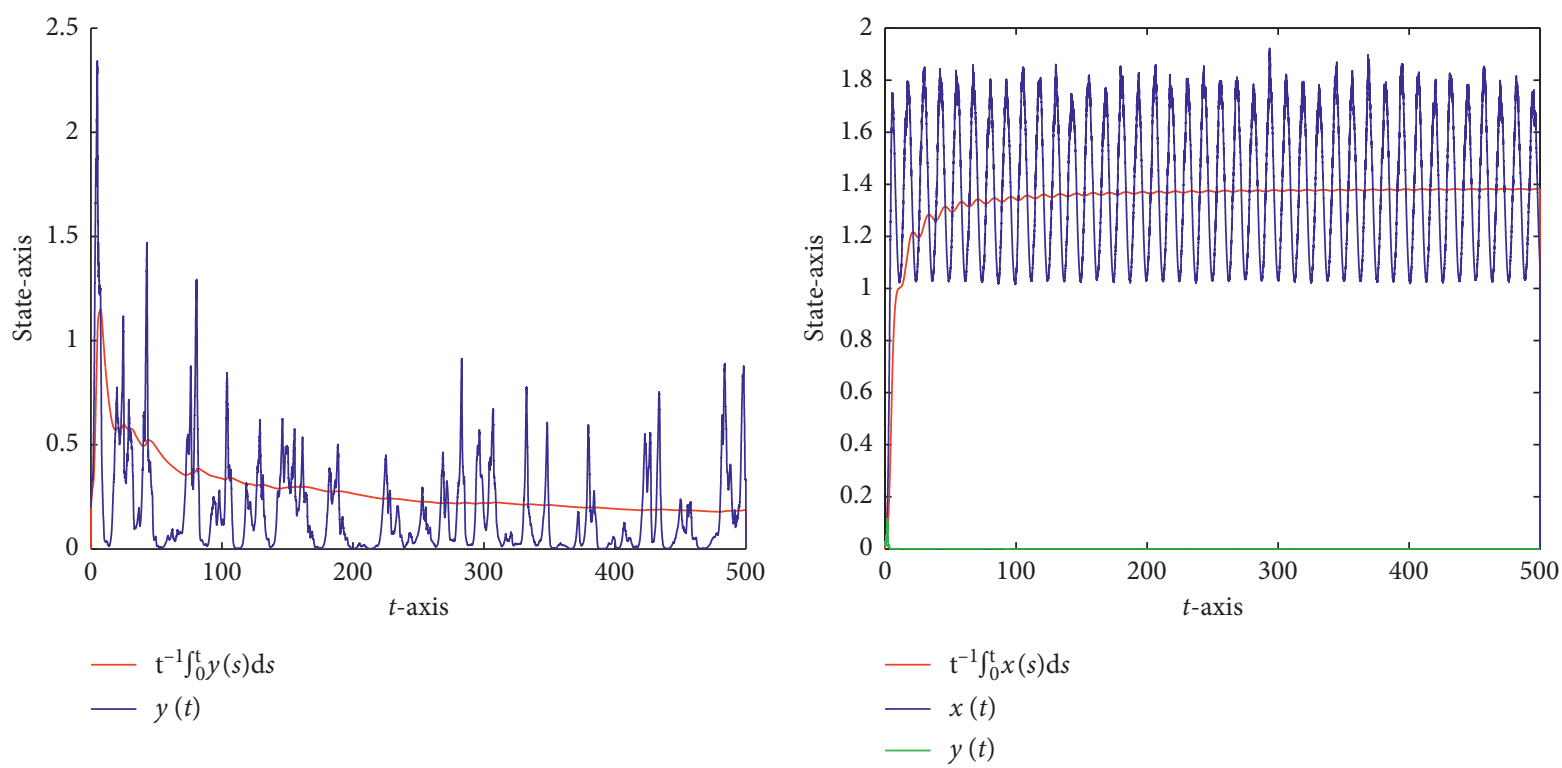

(a)

(b)

Figure 5: The dynamics of $x$ and $y$ of (5) with $n=1$ and $x(0)=y(0)=0.02$. (a) The permanence in the mean of $y$ with $\sigma_{1}(t)=1+0.02 \sin (t / 2), \sigma_{2}(t)=0.01+0.01 \sin (t / 2)$. (b) The permanence in the mean of $x$ and extinction of $y$ with $\sigma_{1}(t)=0.02+0.02 \sin (t / 2), \sigma_{2}(t)=1+0.01 \sin (t / 2)$.

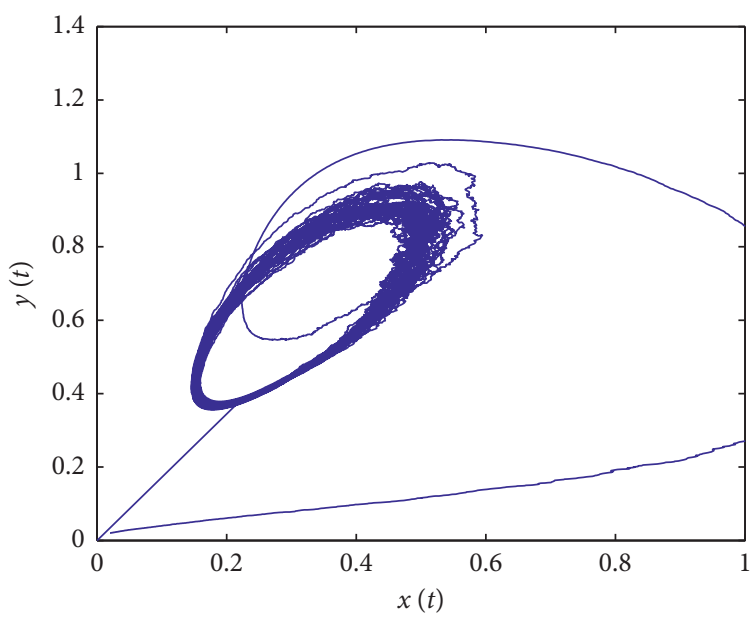

- Stochastic case

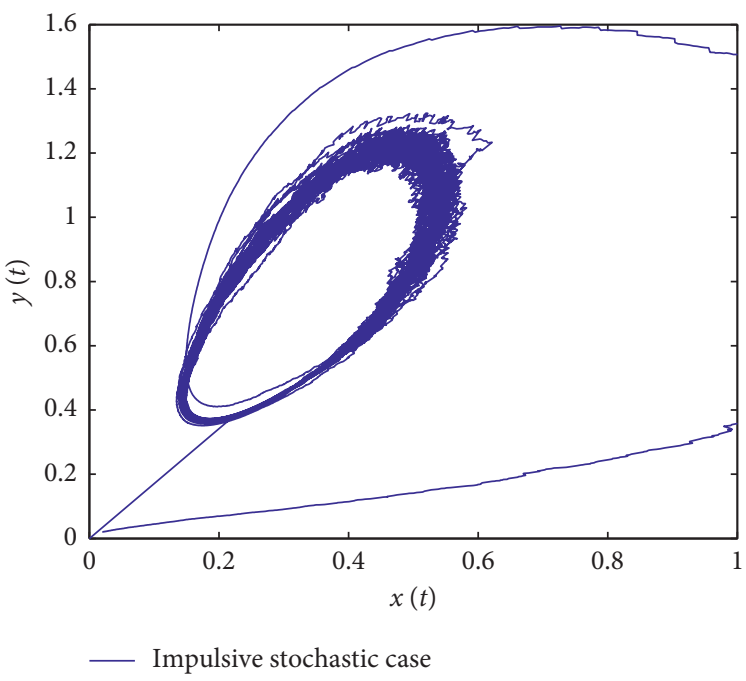

(b)

Figure 6: Continued. 


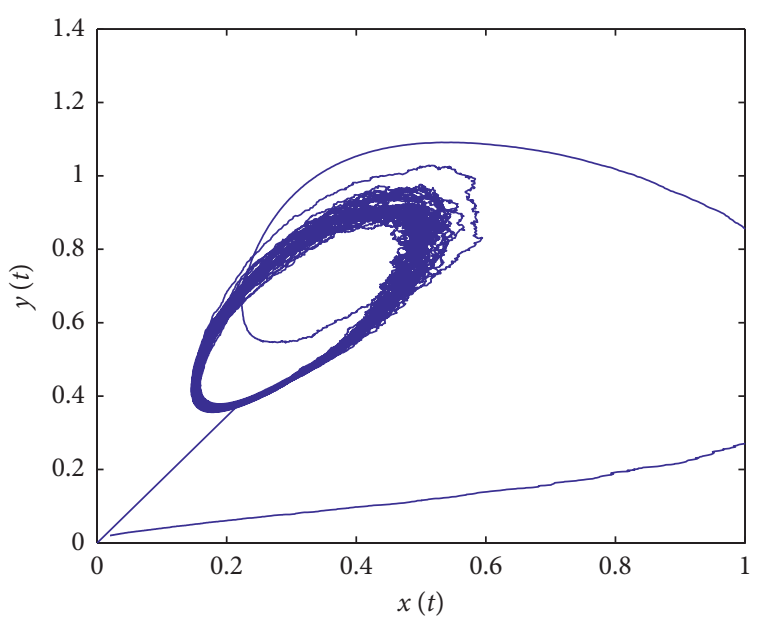

— Stochastic case

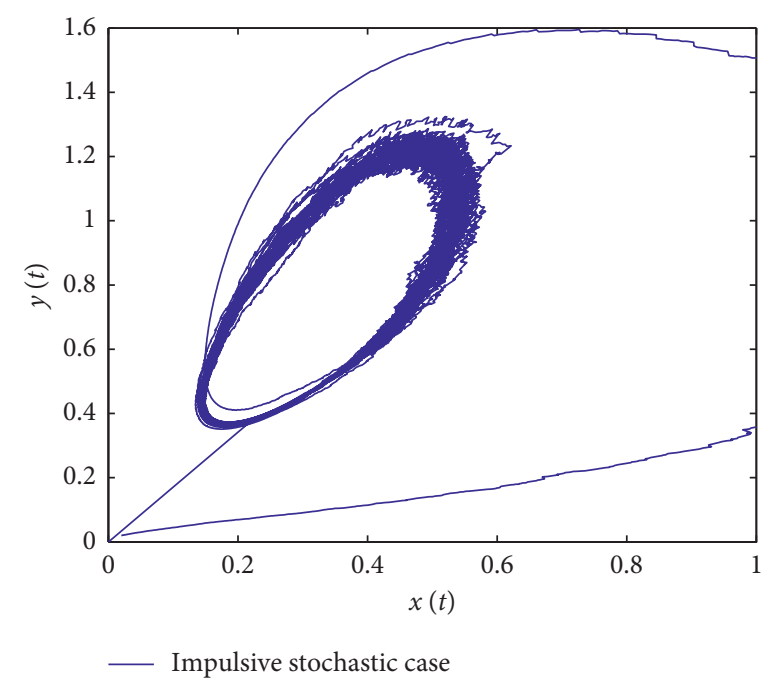

(d)

Figure 6: Periodic Markovian process of (5) with $n=2$ and $x(0)=y(0)=0.02$. (a) The phase graph of deterministic system $\left(\xi_{i k}=0, \sigma_{i}(t)=0, i=1,2\right)$. (b) The phase graph of impulsive system $\left(\sigma_{i}(t)=0, i=1,2\right)$. (c) The phase graph of stochastic system $\left(\sigma_{i}(t)=0, i=1,2\right)$. (d) The phase graph of stochastic impulsive system with $\sigma_{1}(t)=0.02+0.02 \quad \sin (t / 2), \quad \sigma_{2}(t)=0.01$ $+0.01 \sin (t / 2), \xi_{1 k}=0.01+0.01 \sin (t / 2), \xi_{2 k}=0.01+0.01 \sin (t / 2), t_{k}=0.1 k, k \in N$.

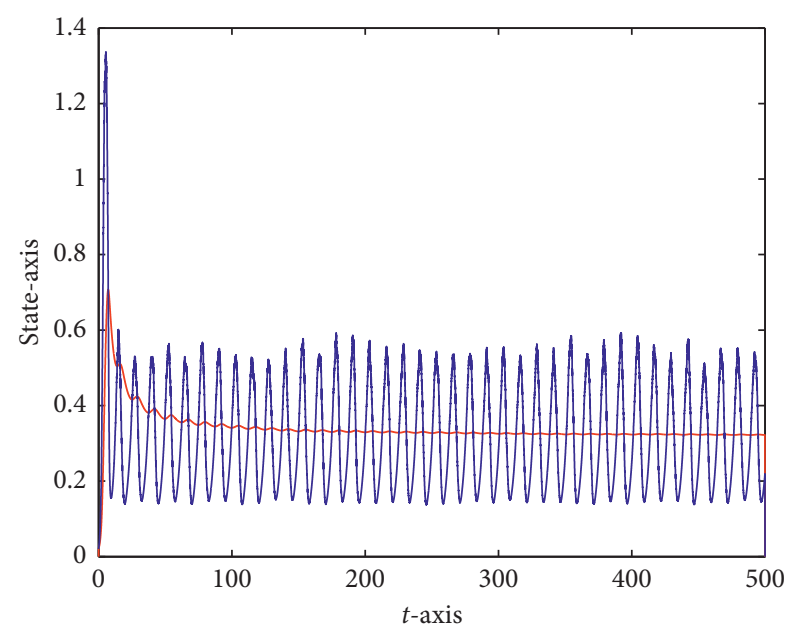

$-\mathrm{t}^{-1} \int_{0}^{\mathrm{t}} x(s) \mathrm{d} s$

$-x(t)$

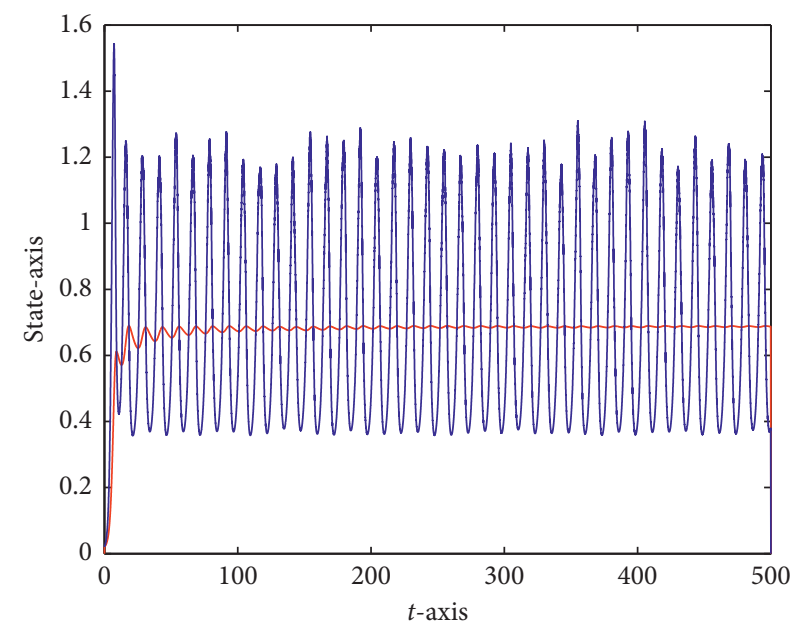

$-\mathrm{t}^{-1} \int_{0}^{\mathrm{t}} y(s) \mathrm{d} s$

$y(t)$

(a)

(b)

Figure 7: Permanence in the mean of (5) with $n=2$ and $x(0)=y(0)=0.02$. (a) The permanence in the mean of $x$. (b) The permanence in the mean of $y$. 


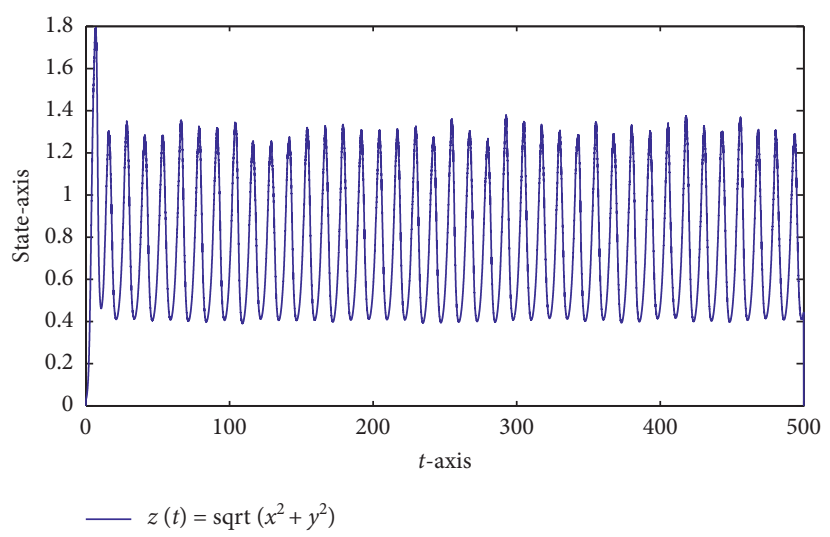

FIGURE 8: Stochastic persistence of (5) with $n=2$ and $x(0)=y(0)=0.02$.

\section{Conclusions and Discussion}

In this paper, we study a stochastic predator-prey system with impulsive effects and Holling type II or Holling type IV functional responses, which contains many models such as those in $[16,23]$. Theorem 1 gives the sufficient conditions of the existence of periodic Markovian process. Theorem 2 represents the extinction and permanence in the mean of predator and prey species. Theorem 3 shows the stochastic permanence of this system. Finally, by writing Matlab codes, some simulations (Figures 1 and 8 ) are provided to verify the main results. Our numerical examples reveal that impulsive and stochastic factors bring much influence to the dynamics of this system.

By comparison analysis, we give Remarks 3 and 5 to show that our main results improve or generalize the corresponding results in $[16,23]$. We apply stochastic analysis techniques instead of constructing some suitable functionals to study the stochastic permanence, which is less applied and relatively new in some sense. In the process of our analysis, Holling-type functional responses bring some difficulties, and we apply inequality techniques to overcome them. In view of too many kinds of functional responses, then how to deal with other functional response such as BeddingtonDeAngelis type? Further, time delays often appear in biological models, and how to discuss the effect of time delays? All these are necessary and very interesting for us to study in the future.

\section{Data Availability}

No data were used to support this study.

\section{Conflicts of Interest}

The authors declare that there are no conflicts of interest regarding the publication of this paper.

\section{Acknowledgments}

This work was supported partially by Natural Science Foundation of China (Grant nos. 11861027 and 11965014) and Inner Mongolia Natural Science Foundation (Grant no. 2018MS01017).

\section{References}

[1] H. Freedman, Deterministic Mathematical Models in Population Ecology, Marcel Dekker, New York, NY, USA, 1980.

[2] S.-B. Hsu and T.-W. Huang, "Global stability for a class of predator-prey systems," SIAM Journal on Applied Mathematics, vol. 55, no. 3, pp. 763-783, 1995.

[3] P. H. Leslie, "Some further notes on the use of matrices in population mathematics," Biometrika, vol. 35, no. 3-4, pp. 213-245, 1948.

[4] P. H. Leslie and J. C. Gower, "The properties of a stochastic model for the predator-prey type of interaction between two species," Biometrika, vol. 47, no. 3-4, pp. 219-234, 1960.

[5] E. C. Pielou, An Introduction to Mathematical Ecology, WileyInterscience, New York, NY, USA, 1969.

[6] Y. Li and D. Xiao, "Bifurcations of a predator-prey system of Holling and Leslie types," Chaos, Solitons \& Fractals, vol. 34, no. 2, pp. 606-620, 2007.

[7] R. Z. Khas'Minskii, Stochastic Stability of Differential Equations, Sijthoff Noordhoff, Alphen Aan Den Rijn, Netherlands, 1980.

[8] W. Zuo, D. Jiang, X. Sun, T. Hayat, and A. Alsaedi, "Longtime behaviors of a stochastic cooperative Lotka-Volterra system with distributed delay," Physica A: Statistical Mechanics and its Applications, vol. 506, pp. 542-559, 2018.

[9] K. Tran and G. Yin, "Stochastic competitive Lotka-Volterra ecosystems under partial observation: feedback controls for permanence and extinction," Journal of the Franklin Institute, vol. 351, no. 8, pp. 4039-4064, 2014.

[10] X. Li and X. Mao, "Population dynamical behavior of nonautonomous Lotka-Volterra competitive system with random perturbation," Discrete \& Continuous Dynamical Systems-A, vol. 24, no. 2, pp. 523-545, 2009.

[11] X. Mao, G. Marion, and E. Renshaw, "Environmental Brownian noise suppresses explosions in population dynamics," Stochastic Processes and their Applications, vol. 97, no. 1, pp. 95-110, 2002.

[12] N. H. Du and V. H. Sam, "Dynamics of a stochastic LotkaVolterra model perturbed by white noise," Journal of Mathematical Analysis and Applications, vol. 324, no. 1, pp. 82-97, 2006.

[13] D. Jiang, Q. Zhang, T. Hayat, and A. Alsaedi, "Periodic solution for a stochastic non-autonomous competitive Lotka- 
Volterra model in a polluted environment," Physica A: Statistical Mechanics and its Applications, vol. 471, pp. 276-287, 2017.

[14] L. Chen and F. Chen, "Dynamic behaviors of the periodic predator-prey system with distributed time delays and impulsive effect," Nonlinear Analysis: Real World Applications, vol. 12, no. 4, pp. 2467-2473, 2011.

[15] Y. Zhao, S. Yuan, and T. Zhang, "Stochastic periodic solution of a non-autonomous toxic-producing phytoplankton allelopathy model with environmental fluctuation," Communications in Nonlinear Science and Numerical Simulation, vol. 44, pp. 266-276, 2017.

[16] D. Jiang, W. Zuo, T. Hayat, and A. Alsaedi, "Stationary distribution and periodic solutions for stochastic HollingLeslie predator-prey systems," Physica A: Statistical Mechanics and its Applications, vol. 460, pp. 16-28, 2016.

[17] Y. Shao and Y. Li, "Dynamical analysis of a stage structured predator-prey system with impulsive diffusion and generic functional response," Applied Mathematics and Computation, vol. 220, pp. 472-481, 2013.

[18] R. Wu, X. Zou, and K. Wang, "Asymptotic behavior of a stochastic non-autonomous predator-prey model with impulsive perturbations," Communications in Nonlinear Science and Numerical Simulation, vol. 20, no. 3, pp. 965-974, 2015.

[19] M. Liu and K. Wang, "Asymptotic behavior of a stochastic nonautonomous Lotka-Volterra competitive system with impulsive perturbations," Mathematical and Computer Modelling, vol. 57, no. 3-4, pp. 909-925, 2013.

[20] S. Zhang and D. Tan, "Dynamics of a stochastic predator-prey system in a polluted environment with pulse toxicant input and impulsive perturbations," Applied Mathematical Modelling, vol. 39, no. 20, pp. 6319-6331, 2015.

[21] S. Zhang, X. Meng, T. Feng, and T. Zhang, "Dynamics analysis and numerical simulations of a stochastic non-autonomous predator-prey system with impulsive effects," Nonlinear Analysis: Hybrid Systems, vol. 26, pp. 19-37, 2017.

[22] M. Liu and K. Wang, "On a stochastic logistic equation with impulsive perturbations," Computers \& Mathematics with Applications, vol. 63, no. 5, pp. 871-886, 2012.

[23] W. Zuo and D. Jiang, "Periodic solutions for a stochastic nonautonomous Holling-Tanner predator-prey system with impulses," Nonlinear Analysis: Hybrid Systems, vol. 22, pp. 191-201, 2016.

[24] Y. Shao, "Globally asymptotical stability and periodicity for a nonautonomous two-species system with diffusion and impulses," Applied Mathematical Modelling, vol. 36, no. 1, pp. 288-300, 2012.

[25] B. G. Zhang and K. Gopalsamy, "On the periodic solution of $\mathrm{N}$-dimensional stochastic population models*," Stochastic Analysis and Applications, vol. 18, no. 2, pp. 323-331, 2000.

[26] D. $\mathrm{Li}$ and D. Xu, "Periodic solutions of stochastic delay differential equations and applications to logistic equation and neural networks," Journal of the Korean Mathematical Society, vol. 50, no. 6, pp. 1165-1181, 2013.

[27] Y. Zhang, S. Chen, S. Gao, and X. Wei, "Stochastic periodic solution for a perturbed non-autonomous predator-prey model with generalized nonlinear harvesting and impulses," Physica A: Statistical Mechanics and its Applications, vol. 486, pp. 347-366, 2017.

[28] A. Yagi and T. V. Ton, "Dynamic of a stochastic predator-prey population," Applied Mathematics and Computation, vol. 218, no. 7, pp. 3100-3109, 2011.

[29] C. Ji and D. Jiang, "Dynamics of a stochastic density dependent predator-prey system with Beddington-DeAngelis functional response," Journal of Mathematical Analysis and Applications, vol. 381, no. 1, pp. 441-453, 2011.

[30] H. Qiu, M. Liu, K. Wang, and Y. Wang, "Dynamics of a stochastic predator-prey system with Beddington-DeAngelis functional response," Applied Mathematics and Computation, vol. 219, no. 4, pp. 2303-2312, 2012.

[31] D. J. Higham, "An algorithmic introduction to numerical simulation of stochastic differential equations," SIAM Review, vol. 43, no. 3, pp. 525-546, 2001. 